\title{
A Reappraisal of the Terverticillate Penicillia Using Biochemical, Physiological and Morphological Features I. Numerical Taxonomy
}

\author{
By P. D. BRIDGE, ${ }^{1}$ D. L. HAWKSWORTH, ${ }^{1 *}$ Z. KOZAKIEWICZ, \\ A. H. S. ONIONS, ${ }^{1}$ R. R. M. PATERSON, ${ }^{1}$ M. J. SACKIN ${ }^{2}$ \\ AND P. H. A. SNEATH ${ }^{2}$ \\ ${ }^{1} C A B$ International Mycological Institute, Ferry Lane, Kew, Surrey TW9 3AF, UK \\ ${ }^{2}$ Department of Microbiology, University of Leicester, PO Box 138, Medical Sciences Building, \\ University Road, Leicester LE1 9HN, UK
}

(Received 20 January 1989; revised 6 June 1989; accepted 17 July 1989)

\begin{abstract}
Three-hundred-and-forty-eight strains representing the major species of terverticillate penicillia, and including representatives of other closely and distantly related species, were included in a numerical taxonomic study. One-hundred characters were derived from morphological features, physiological and biochemical activities and SEM micrographs. Strains were compared by both Gower's coefficient and Pattern difference, and clustered using the average linkage algorithm. Thirty-seven species or species-complex clusters were recovered at approximately $70 \%$ similarity; they generally corresponded to existing taxonomic concepts. Several species were shown to contain variants or chemotypes which were often supported by differences in conidial shape and ornamentation. The use of different types of characters enabled a number of new and previously accepted species to be shown to be either variants or deteriorated examples of other species. Variation in properties both between and within species was considered, particularly in relation to strain stability.
\end{abstract}

\section{INTRODUCTION}

The generic name Penicillium was introduced by Link (1809), and the species $P$. expansum is now accepted as the lectotype for the genus (Hawksworth, 1986). The genus has been divided into sections or subgenera based on the number and arrangement of branches of the sporebearing structures, the conidiophores (e.g. Thom, 1930; Pitt, 1980). Despite many studies and monographs (e.g. Dierckx, 1901; Westling, 1911 ; Biourge, 1923; Thom, 1930; Raper \& Thom, 1949; Abe, 1956; Samson et al., 1976; Fassatiova, 1977; Pitt, 1980), the taxonomy of the section of the genus containing cultures with three or more levels of branches remained unsatisfactory until relatively recently (Onions et al., 1984; Samson \& Gams, 1984). The species concepts and names adopted for these fungi have not always been consistent between authors, making identifications of cultures and the interpretation of literature reports difficult (Onions et al., 1984; Samson \& Gams, 1984). Another problem is the poor condition of some historically and nomenclaturally important strains in culture, and their generally unremarkable morphological features. Previous taxonomic studies on the terverticillate penicillia have been based almost solely on morphology and gross physiology, and results from more recent biochemical studies have suggested that new species and chemotypes may be necessary to characterize accurately these organisms (Frisvad, 1985; Pitt \& Hawksworth, 1986). Since the introduction of a multidisciplinary approach to the taxonomy of Penicillium (Abe, 1956; Frisvad, 1981; Bridge et $a l ., 1986)$ and the start of the present investigation in 1984, several further studies have considered the application of particular new techniques within specific areas of the genus. Additional techniques have been applied in the delimitation of Penicillium species in recent 
years, such as physiological criteria (Pitt, 1973; Bridge, 1985; Frisvad, 1985), secondary metabolite production (Ciegler et al., 1973; Frisvad, 1986; El-Banna et al., 1987), serology (Polonelli et al., 1986), isozyme electrophoresis (Cruickshank \& Pitt, 1987), pyrolysis-GLC (Söderström \& Frisvad, 1984), and cross-point separation (Ström, 1986). However, in most cases the new techniques have been used in isolation from each other and some of the deteriorated or markedly atypical strains were not examined.

In general, these latter studies have involved either a large number of strains from a few species, or relatively few strains from a number of species, and have rarely been subsequently checked for reproducibility. The reproducibility and applicability of such approaches was therefore in urgent need of evaluation before such characters became regarded as diagnostic criteria.

The three branched penicillia have been variously grouped under the terms 'Asymmetrica subsection Fasciculata'(Raper \& Thom, 1949), 'biverticillate penicillia'(Samson et al., 1976) and 'subgenus Penicillium' (Pitt, 1980). Further subdivisions within these taxa have been proposed according to colony texture (e.g. Raper \& Thom, 1949) and further details of the branching pattern (Pitt, 1980). Species were originally delineated on the basis of colony morphology, diameter and pigmentation; conidiophore shape and ornamentation; features of the conidiogenous cells; and conidium shape, colour and ornamentation (e.g. Westling, 1911; Raper $\&$ Thom, 1949). More recent studies have included some physiological characters such as growth temperatures and growth on nitrite as well as the production of specific secondary metabolites or isozyme electrophoresis (e.g. Abe, 1956; Ciegler et al., 1973; Pitt, 1973; Frisvad, 1981 ; Frisvad \& Filtenborg, 1983; Cruickshank \& Pitt, 1987).

Numerical taxonomy based on a mixture of morphological, physiological and biochemical characters, followed by an objective numerical analysis has rarely been attempted in filamentous fungi (Sneath, 1985), and those that have been done have largely been restricted to fewer than 100 cultures, often to help clarify relationships in particular host- or substrate-related situations (e.g. Mueller, 1985; Mordue et al., 1989). Others have not proved entirely satisfactory (e.g. Kendrick \& Proctor, 1964).

This paper reports the results of an integrated multidisciplinary study on the terverticillate penicillia, combining characters from morphology, ultrastructure, physiology and biochemistry with critical studies on strain and test variation and numerical taxonomic analysis.

\section{METHODS}

Strains studied. The 348 strains studied are given in Table 1. The names given to individual strains were initially those assigned to the cultures by the donors, and apart from checking for purity, initial identification was not carried out on receipt. Strains were maintained during the study on stock slopes of Czapek-Dox agar $(\mathrm{Cz}$; Smith \& Onions, 1983) preserved at $-20^{\circ} \mathrm{C}$. All strains were grown aerobically at $25^{\circ} \mathrm{C}$ unless stated otherwise.

Inocula. Strains were transferred and inoculated as conidial suspensions. Inoculation of plates for morphological testing and SEM studies was by conidia suspended in a semisolid agar/Tween 80 medium (Pitt, 1980). Inoculation of plates and bottles for physiological testing was by conidia suspended in aqueous Tween $80(0.02 \%$; Bridge, 1985).

Test selection. One-hundred characters (Table 2) were selected from more than 200 evaluated in previous studies with smaller sets of strains (Bridge et al., 1986, 1987; Paterson, 1986). Tests or procedures that had been shown to be irreproducible, or had appeared of little value in earlier studies were not used.

Colony and cellular morphology. Duplicate plates of $\mathrm{Cz}$ agar were three-point-inoculated with a sterile wire as described in Pitt (1980) and incubated for $7 \mathrm{~d}$. Colony characters were recorded directly from these plates with the aid of a binocular dissecting microscope. Slides were prepared in warmed lactophenol cotton blue. The terminology for morphological structures used is that of Minter et al. (1986).

Physiological characters. Growth at different temperatures on $\mathrm{Cz}$ agar, growth on tannic acid, growth and base production on lactic acid, citric acid, Tween 80 and urea, and hydrolysis of casein, gelatin, RNA, DNA, starch and cellulose were determined as described by Bridge (1985). In addition, growth on acetic acid agar, growth and basic reaction on creatine agar and growth on nitrite-sucrose agar (Frisvad, 1981), and the ability to produce yellow colonies on ammonium oxalate (CZNH4 agar; Bridge et al., 1986) were assessed.

Secondary metabolites. The production of secondary metabolites on Czapek Yeast agar (CYA; Pitt, 1980) and Yeast Extract Sucrose agar (YES; Davis et al., 1966) was detected by TLC with the agar plug method of Paterson (1986), adapted from the methods of Frisvad (1981). 
Table 1. Strains studied arranged in cluster order $\left(S_{\mathrm{G}}\right.$ coefficient $)$

IMI no.

183170

291201

297902

293185

204208

296937

286763

$169087 \mathrm{a}$

40218

39811

297980

285507

68234

\section{IMI}

Comments

no.
Comments

Single-member cluster (SM1)

91944iii ex-neotype of $P$.

$$
\text { glabrum }
$$

Cluster 1 ( $P$. expansum)

297898

297899

284419

207082

Cluster 2 ( $P$. expansum)

246656

Cluster 3 ( $P$. expansum)

299046

Cluster 4 ( $P$. aurantiogriseum)

$$
\text { ex-type of } P \text {. cyclopium }
$$

2

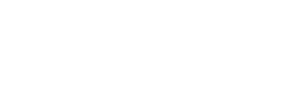

\section{ex-type of $P$.} carneolutescens

ex-type of $P$. roquefortii
var. punctatum

297547

39761 ex-neotype of $P$.

297908 expansum

92199

ex-type of $P$.

brunneoviolaceum

89374

291195

34913

ex-type of $P$.

glaucocoeruleum

ex-neotype of $P$.

puberulum

40211

297890

297953

286106

195050

195050

\section{ex-neotype of $P$.}

aurantiogriseum aurantiogriseum

Cluster 5.1 ( $P$. solitum var. solitum)

291199

$$
\text { ex-type of } P \text {. }
$$

291198

297556

293201

Cluster 5.2 ( $P$. solitum var. solitum)

$\begin{array}{rr}92225 & \\ 68235 & \begin{array}{r}\text { ex-type of } P . \\ \text { pseudocasei }\end{array} \\ 293179 & \\ 194737 & \begin{array}{r}\text { ex-type of } P . \\ \text { flavoglaucum }\end{array}\end{array}$

Cluster 5.3 ( $P$. solitum var. crustosum)
92224

224935

174158

265302

39803

92235

243002

297909

284413

286084

291555

286094

280215

285516

297910

285515

24318

297951

297957

297969

291543

89386

265297

262790

91939

89385

39808

143321

28040
IMI

no.

Comments ex-type of $P$. aurantiogriseum var. poznaniensis

ex-type of $P$. schneggii
91939

293182

243053 
Table 1 (continued)

IMI

no.

52736

281775

89378

206416

209783

287746

293210

291196

92276

39810 ex-type of $P$. solitum

34911 ex-type of $P$. gladioli

$\begin{array}{cc}291197 & \begin{array}{c}\text { ex-type of } P \text {. casei var. } \\ \text { compactum }\end{array} \\ 92219 \quad \begin{array}{c}\text { ex-neotype of } P . \\ \text { griseobrunneum }\end{array} \\ 39766 & \end{array}$

286235

291191

ex-type of $P$.

janthogenum

24314

ex-type of $P$.

chrysogenum

285508

40028

ex-type of $P$. echinulatum

68236 ex-type of $P$. palitans var. echinoconidium

166526(B)

40223
IMI

no.

Comments

IMI

no.

Comments

Cluster 5.4 ( $P$. solitum var. crustosum)

181050

296938

281551

293196

Cluster 5.5 ( $P$. solitum var. solitum)

154731

321495

291547

291192

297999

351512

214070

51355

49096

Cluster 5.6 ( $P$. solitum var. solitum)

297956

298007

Cluster 5.7 ( $P$. solitum var. solitum)

$\begin{array}{lll}39822 & \text { ex-type of } P . & 297997 \\ & \text { lanosogriseum } & 321496 \\ 39820 & \text { ex-type of } P \text {. biforme } & 92264 \\ 297892 & & 40215 \\ 321523 & \text { ex-type of } P . & \\ & \text { majusculum }\end{array}$

ex-type of $P$. olivicolor ex-type of $P$. palitans

ex-type of $P$

verrucosum var.

melanochlorum

SM2

285531

SM3

192904 ex-type of $P$. psittacinum

\section{SM4}

39819 ex-type of $P$ lanosoviride

\section{SM5}

89312 ex-type of $P$. cyclopium var. album

Cluster 6 ( $P$, aurantiogriseum)

$\begin{array}{ccc}34913 & \begin{array}{c}\text { ex-neotype of } P . \\ \text { puberulum }\end{array} & 92222 \\ & 297894\end{array}$

Cluster 7 (weak $P$. chrysogenum)

91935

$39762 \mathrm{iii}$

Cluster 8 ( $P$. echinulatum)

68236 ex-type of $P$. palitans

Cluster 9 ( $P$. olivinoviride) 
Table 1 (continued)

IMI

no. Comments

298083

ex-neotype of $P$ granulatum

285512

246204

296066

264173

291539

37767

91020(b)

297895

75832

ex-neotype of $P$. griseofulvum

IMI

Comments

IMI

no.

Comments

Cluster 10 ( $P$. granulatum var. globosum) 297543

299049

Cluster 11 ( $P$. granulatum var. granulatum) 321513

297543

Cluster 12 (P. hirsutum) 196570

Cluster $13(P$. hordei)

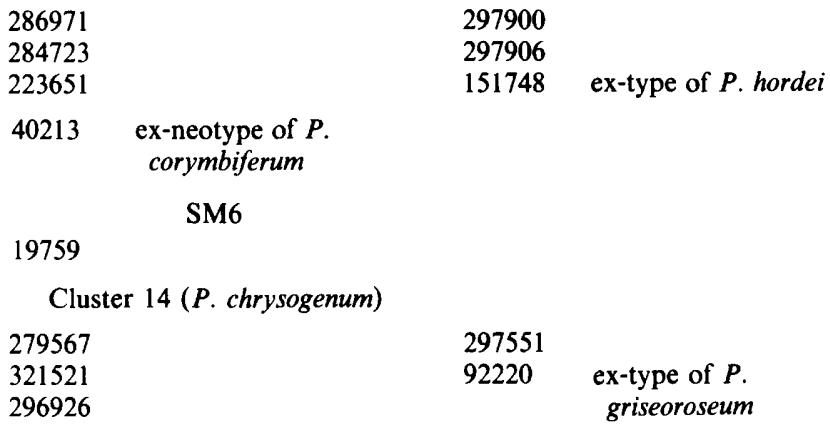

Cluster 15 ( $P$. glandicola var. mononematosa)

296925
321492
321493

ex-type of $P$. 321494

viridicatum var. $\quad 321509$

verrucosoidis 296930

321508

39767

297544

ex-type of $P$. nigricans

Cluster 16 ( $P$. camembertii)

91924(A)

285506

129208

287746

ex-type of mutant $P$. mali

Cluster 17 (Penicillium sp.)

28810

ex-type of $P$. caseicola

27831

ex-type of $P$.
camembertii

Cluster $18(P$. verrucosum)

285522

284410

297897

260915

285523

295238

297896

284409

(2)

Cluster 19 ( $P$. atramentosum)

210922

285530

295234

210922

298080

Cluster 20 ( $P$. atramentosum)

200310 ex-neotype of $P$. verrucosum

Cluster 21 ( $P$. atramentosum) 
P. D. BRIDGE AND OTHERS

Table 1 (continued)

IMI

no.

321510

321500

280297

17456

17456

39824

94149

286753

ex-type of $P$.

stoloniferum

Comments

no.

Comments

Cluster 22 (Penicillium sp.)

321501

SM7

285510

\section{SM8}

296073

SM9

134650 ex-type of $P$.

majusculum

Cluster 23 ( $P$. citrinum)

92196 ex-type of $P$. citrinum

Cluster 24 ( $P$. brevicompactum)

92044

285220

40225

pe of $P$.

brevicompactum

321514

Cluster 25 ( $P$. raistrickii)

40221

68414

Cluster 26 ( $P$. aurantiogriseum var. neoechinulatum)

85513

ex-type of $P$.

296927

321490

discolor

$92209 \mathrm{ii}$

ex-type of $P$.

subcinereum,

ex-neotype of $P$.

citreonigrum

297546

293178

Cluster 27 ( $P$. viridicatum)

39758ii ex-neotype of $P$.

viridicatum

173209

284408

265301

293186

285525

205652

297893

297901

174718 ex-type of $P$. conservandi
150406

284414

192905

297907

293194

293197

Cluster 28 ( $P$. viridicatum)

293187

297904

291201

Cluster 29 ( $P$. griseofulvum)

39809 ex-type of $P$. urticae

297542

28808

291549

ex-type of $P$. patulum

92273

296934

296934

285527

Cluster 30 (Penicillium sp.)

284415

192216

92215
MI

no.

Comments

21511 
Table 1 (continued)

IMI

no.

Comments

297905

297911

299048

321502

34846

ex-type of $P$.

aurantiovirens

285518

285509

291202

39754

$$
\begin{aligned}
& \text { ex-neotype of } P \text {. } \\
& \text { corylophilum }
\end{aligned}
$$

213846

39739

181051

39752

297966

192332

285528

44744

39754
IMI

no.
IMI

no.

Comments

Cluster 31 ( $P$. hirsutum var. allii)

321505

Cluster 32 (coremial isolates)

321504

321520

297546

Cluster 33 ( $P$. aurantiogriseum var. melanoconidium)

321503

39759 ex-type of $P$. notatum

Cluster 34 ( $P$. clavigerum)

$39807 \quad$ ex-neotype of $\boldsymbol{P}$. clavigerum

Cluster 35 ( $P$. roquefortii)

$148775 \quad 129207$

24313 ex-type of $P$. roquefortii 293190

Cluster 36 ( $P$. corylophilum)

39754 ex-neotype of $P$. 296936 corylophilum

$297991 \quad 204081$

$90178 \quad 297548$

$285455 \quad 291193$

29700

40238 ex-neotype of $P$.

janthinellum

197477 ex-neotype of Talaro-

myces flavus

39733

ex-neotype of $P$.

verrucosum var.

verrucosum

ex-type of $P$. javanicum

Cluster 37 ( $P$. claviforme)

40237 ex-neotype of $P$.

194564

40237 ex-neotype of $P$.

claviforme

SM11

193019 ex-neotype of $P$.

funiculosum

Scanning electron microscopy. SEM of 21-d-old cultures (to ensure mature conidia) from $\mathrm{Cz}$ agar was done using a cryo-stage as described in Kozakiewicz (1989). In addition to the conidial shape and ornamentation characters, the length and breadth of conidia were determined from the SEM micrographs with a MADS digitizer.

Coding of results. Missing or inapplicable results (such as base production on carbon source when growth on carbon source was negative) were coded as 'no comparison' (NC). Physiological characters were coded as 0 for no reaction, 1 for a weak (but definite) reaction and 2 for a strong reaction. The one exception was production of yellow colonies on CZNH4 agar, which was coded as 0 for negative and 1 for positive.

Morphological characters were coded as binary with the following exceptions: colony diameter, first-level branch length and conidiogenous cell length were recorded as the mean measurement from 20 samples and a coefficient of variation was determined from these measurements; roughening of the conidiophore on a scale from 0 for smooth to 2 for very rough; colony profile as 1 for flat and spreading, 2 for raised and 3 for high domed; fasciculation on a scale from 0 for not fasciculate up to 3 for very fasciculate and 4 being used only when coremia 
were produced. Characters relating to colour were recorded on an 'all present' principle so that a colony with greygreen conidia in the centre and blue-green conidia at the margin was recorded as both blue-green and grey-green. This principle was also used with conidium shape.

Coding of secondary metabolite data was primarily determined by the reliability and reproducibility of the detection. Metabolite results seen in at least $67 \%$ of all replicates were coded as 2 , and $83 \%$ of these were $100 \%$ reproducible; metabolite results seen in $50 \%$ of replicates were coded as 1 , and all other metabolite results were coded as 0 .

Reproducibility. Strain reproducibility was tested by subculturing duplicate lines of 10 strains on receipt. These duplicate cultures were maintained and tested separately. Test reproducibility was examined from all tests for each set of data; physiological tests and secondary metabolite screening were repeated for 148 strains; morphology tests were repeated for 52 strains; and SEM tests were repeated for 227 strains. Tests were repeated between 12 and 18 months after the original tests. For comparison, multistate characters were converted to binary $(0=-v e, 1$ or $2=+v e$ ). Standard deviations of measurements suggested that $\pm 2 \mathrm{~mm}$ was a suitable cutoff for comparisons of colony diameters.

Numerical analyses. All computation was done on a DEC VAX cluster through the TAXPAK numerical taxonomy package (M. J. Sackin, unpublished) at the Microbiology Department, Leicester University, UK). Resemblances between strains were calculated with Gower's coefficient $\left(S_{\mathrm{G}}\right.$; cf. Sneath \& Sokal, 1973) and the Pattern coefficient $\left(D_{\mathrm{p} 2} ;\right.$ Sackin, 1981). Corresponding vigour values and differences in vigour were also calculated $\left(D_{\mathrm{v} 2}\right.$; Sackin, 1981). In both cases all matching negative results were excluded from calculations in order to avoid excessive weighting where there were multiple characters relating to a single phenomenon, such as colony colour. Clustering was by average linkage (UPGMA; Sneath \& Sokal, 1973) and dendrograms were plotted. Overlap between adjacent pairs of taxa was estimated with the TESTDEN program which determined overlap between adjacent groups on the same arm of the dendrogram (Sneath, 1979). In cases where there were four or more degrees of freedom, the apparent overlap was compared for significance against a critical overlap set at both $2.5 \%$ and that expected for a rectangular distribution, as described in Sneath (1979). Principal coordinates analysis was done by the method of Gower (1966), with a distance matrix calculated by a transformation of the $S_{\mathrm{G}}$ values $\left[d=\left(1-S_{\mathrm{G}}\right)^{1 / 2}\right)$, using a program written by M. J. Sackin (unpublished).

\section{RESULTS AND DISCUSSION}

\section{Reproducibility and other analyses}

All duplicate strains were recovered in the same clusters as their parental strains with two exceptions (IMI 34913 and IMI 291199), which were found to be either very deteriorated or contaminated at the end of the study. The average similarity for the eight remaining pairs in the Gower's coefficient dendrogram was 0.82 , a low figure for numerical taxonomy studies (e.g. Williams et al., 1983; Bridge \& May, 1984). The discounting of matching negative results has resulted in generally low similarities throughout the study. Variation in phenotypic properties (often seen as sectoring) within single strains of conidial fungi is well known (e.g. Burnett, 1976; Klittich et al., 1988) and phenotypic variation within some of the strains of Penicillium used in this study has been reported (Bridge et al., 1987). Test duplication after more than 12 months maintenance gave $4.4 \%$ discrepancy for physiological and SEM characters and $7.6 \%$ discrepancy for morphological characters. These figures were considered acceptable in this study in view of the inherent genetic variability and the subjectivity often involved in scoring morphological characters such as shades of colour.

Reproducibility for secondary metabolite production was not entirely satisfactory. Duplicated cultures grown, extracted and tested together very rarely showed discrepancies. However, when individual strains were screened over a period of time, not all metabolites were seen during each screening. This type of discrepancy in secondary metabolite production by fungi has been widely reported and may be due to, amongst other things, variations in relative concentrations of metabolites at different times caused by, for example, instability of enzymes involved in the biosynthetic pathways (e.g. Lam et al., 1988). It was for this reason that the final set of secondary metabolites used was restricted to those that were $50-100 \%$ reproducible, and the irreproducibility of the production of a particular metabolite was represented in the scoring of the character, so that their effect on similarity values was minimized when compared to apparent non-producers. Computation of the data without the secondary metabolite results gave a lower value for the cophenetic correlation coefficient and did not improve clustering.

The cophenetic correlation for the dendrogram derived from Gower's coefficient was $0 \cdot 788$. 
This would be a relatively low figure for a numerical taxonomy study on bacteria (Jones \& Sackin, 1980); however, this statistic has rarely been used with mycological data, and so a fuller interpretation is difficult.

Overlap between pairs of adjacent groups on the same arm of the Gower's coefficient dendrogram was investigated and compared to critical overlaps set for that expected for a rectangular distribution and $2.5 \%$. In this dendrogram clusters appeared either singly or in small groups on distinct arms which were subsequently joined by a number of very close links. The overlap results mirrored the lengths of the stems on the dendrogram; comparisons between clusters on the same arm of the dendrogram showed either 95 or $99 \%$ confidence that the groups were distinct. However, comparisons between arms of the dendrogram that were very close together suggested overlap. These results indicate that the species clusters, while representing distinct entities, may be placed either in or just outside a larger indistinct continuum.

\section{Cluster analyses}

A simplified dendrogram based on Gower's coefficient and UPGMA clustering is shown in Fig. 1. Two-hundred-and-ninety-seven strains $(85 \%)$ formed 37 species or species-complex clusters at approximately $70 \%$ similarity. The characteristics of these clusters are given in Table 2. In addition, 20 of the strains included as either single representatives of species or as representatives of dissimilar species formed single member or small, loosely defined clusters. In total $317(91 \%)$ of the strains were considered grouped. The twenty most useful differential tests between clusters were calculated from Gyllenberg's Sum of C (see Sneath \& Sokal, 1973), and are indicated in Table 2. The dendrogram obtained from the Pattern coefficient was broadly similar, although some clusters merged, and this is considered in the following cluster descriptions. Names were assigned to clusters on the basis of the oldest recognized ex-type strain present; in clusters where no ex-type strains were recovered names are based on species descriptions and strains regarded as typical.

\section{Description and identity of clusters}

Single-member cluster 1 (SM1) contained a single strain received as $P$. frequentans ( $P$. glabrum), which had been included as an example of a different section of the genus, subgenus Aspergilloides. The separation of this strain was therefore as expected.

Cluster 1 contained nine strains and agrees well with the species concept of $P$. expansum (Raper \& Thom, 1949; Samson et al., 1976; Fassatiova, 1977; Pitt, 1980). Our culture of the ex-neotype strain of $P$. expansum was not recovered in this cluster, although another line of the ex-neotype tested subsequently showed good agreement in all of the typical characters (following paper: Bridge et al., 1989). The ex-neotype strain of $P$. expansum originally used in this study must therefore be considered aberrant.

Cluster 2 contained three strains received as examples of a possible new species, characterized primarily by secondary metabolite profiles (J. C. Frisvad, personal communication). The group showed considerable similarity with $P$. expansum, although strains grew at $37^{\circ} \mathrm{C}$; however, unlike all except one of the other $P$. expansum strains, these three strains were isolated from tropical or equatorial zones and it would seem most likely that they represent a variant of P. expansum.

Cluster 3 consisted of two strains supplied as freshly isolated strains of $P$. expansum. Both strains were morphologically very similar to cluster 1 , but differed in producing a basic reaction from lactic acid and failing to hydrolyse DNA. These strains represent a variant of $P$. expansum. However, this cluster was moved considerably down the dendrogram in the Pattern representation and so it is unlikely that its separation from cluster 1 is due entirely to the greater vigour of the fresh isolates (see later).

Cluster 4 consisted of 44 strains including 12 ex-type or neotype strains. The cluster contains strains considered representative of $P$. aurantiogriseum, and with the exception of the aberrant $P$. expansum noted above, the remaining ex-type cultures have all been regarded as synonyms of this species (Raper \& Thom, 1949; Samson et al., 1976; Fassatiova, 1977; Pitt, 1980).

Cluster 5 consisted of 77 strains including 16 ex-type cultures. Many of these strains have been 


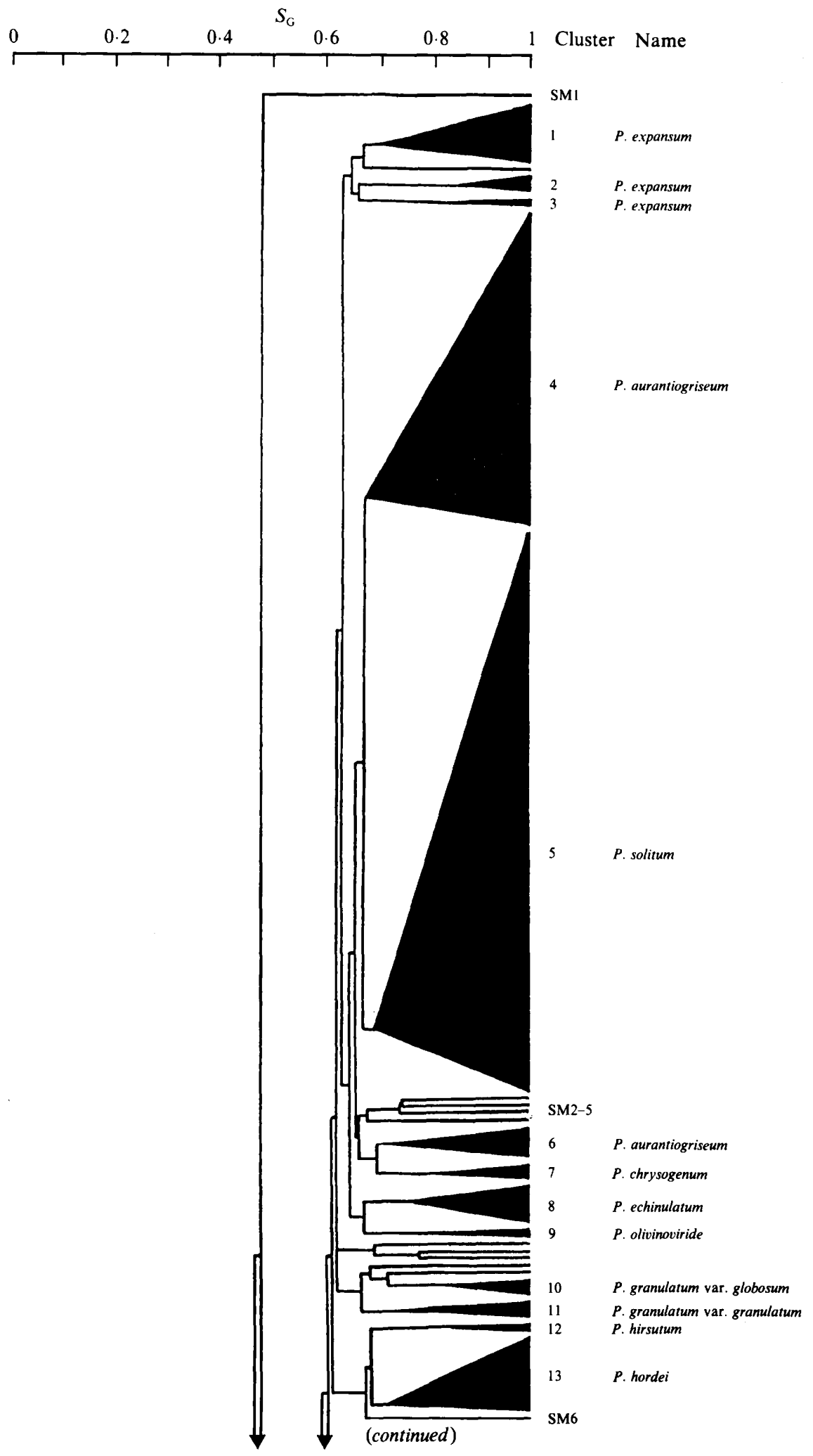




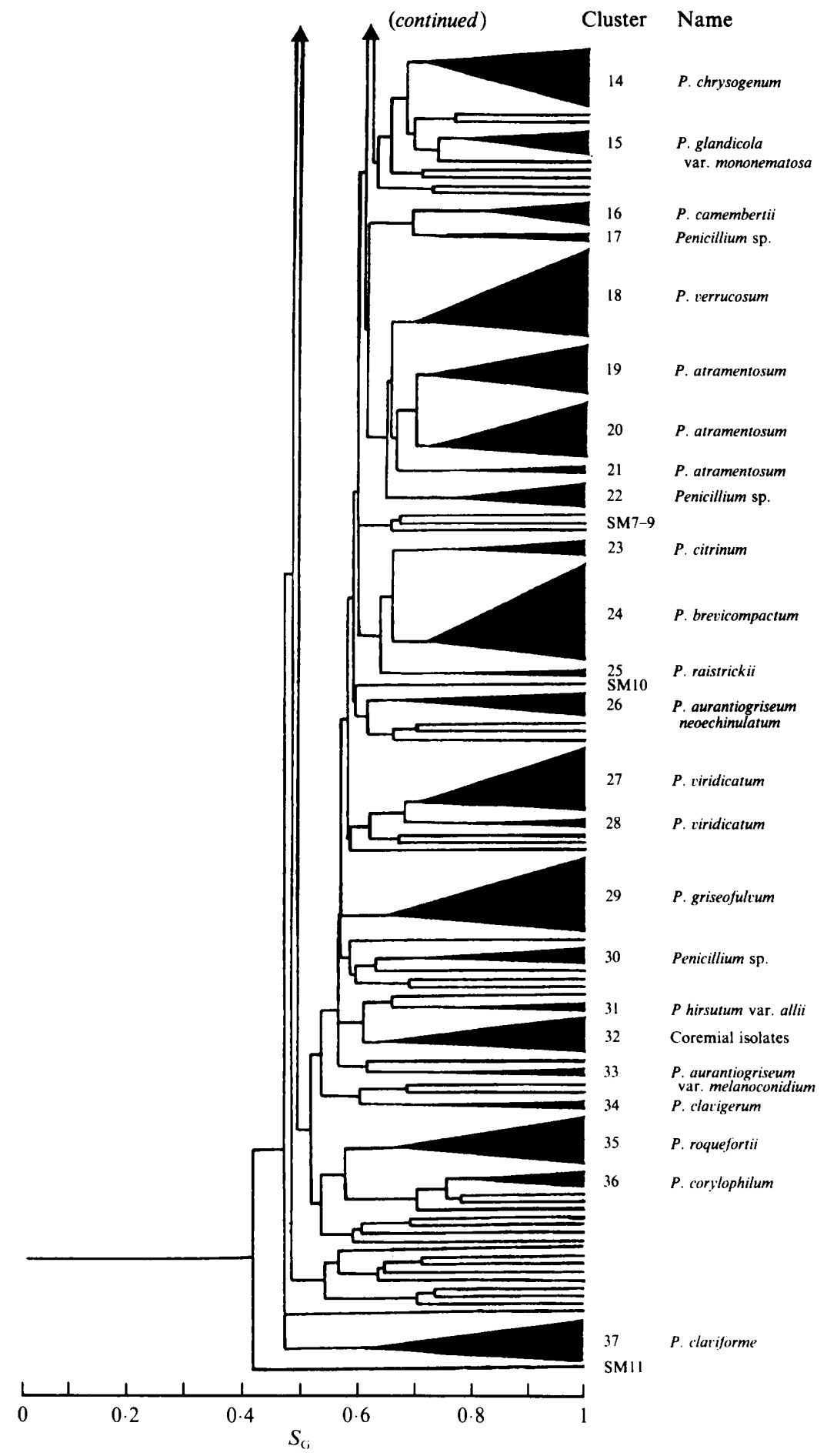

Fig. 1. Simplified dendrogram based on Gower's coefficient. 
Table 2. Distribution of positive characters in clusters

Values are number of strains positive in each cluster. D indicates the 20 most useful diagnostic tests, including fasciculate colonies and coremia production as two characters (test 27).

Cluster

1. P. expansum

2. P. expansum

3. P. expansum

4. $P$. aurantiogriseum

5. P. solitum

6. P. aurantiogriseum

7. P. chrysogenum

8. P. echinulatum

9. P. olivinoviride

10. P. granulatum var. globosum

11. $P$. granulatum var. granulatum

12. P. hirsutum

13. P. hordei

14. P. chrysogenum

15. $P$. glandicola var. mononematosa

16. P. camembertii

17. Penicillium sp.

18. P. verrucosum

19. P. atramentosum

20. P. atramentosum

21. P. atramentosum

22. Penicillium sp.

23. P. citrinum

24. P. brevicompactum

25. P. raistrickii

26. $P$. aurantiogriseum var. neoechinulatum

27. P. viridicatum

28. P. viridicatum

29. P. griseofulvum

30. Penicillium sp.

31. $P$. hirsutum var. allii

32. Coremial strains

33. $P$. aurantiogriseum var. melanoconidium

34. P. clavigerum

35. $P$. roquefortii

36. $P$. corylophilum

37. P. claviforme

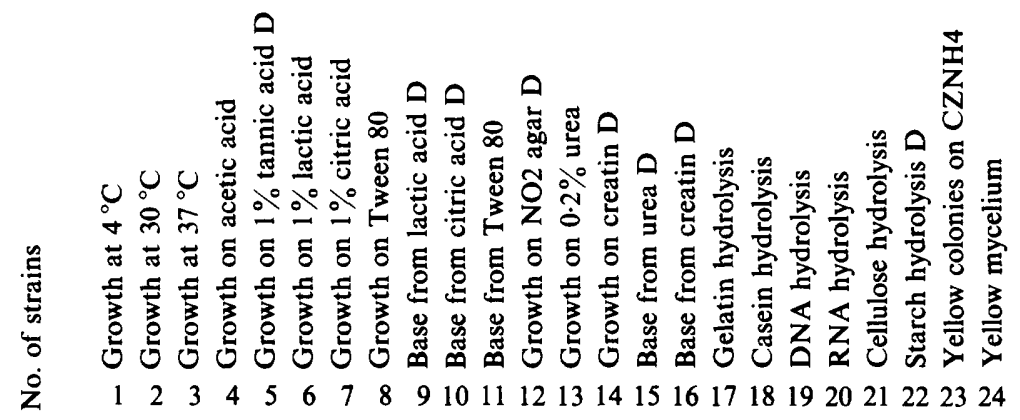

$\begin{array}{llllllllllllllllllllllll}9 & 9 & 0 & 0 & 9 & 9 & 9 & 9 & 0 & 6 & 9 & 0 & 9 & 8 & 0 & 5 & 9 & 8 & 8 & 8 & 1 & 9 & 0 & 0\end{array}$ $\begin{array}{llllllllllllllllllllllll}3 & 3 & 3 & 0 & 3 & 3 & 3 & 3 & 0 & 2 & 3 & 0 & 3 & 3 & 0 & 3 & 3 & 3 & 0 & 1 & 0 & 3 & 0 & 0\end{array}$ $\begin{array}{llllllllllllllllllllllll}2 & 2 & 0 & 0 & 2 & 2 & 2 & 2 & 2 & 2 & 2 & 0 & 2 & 2 & 0 & 2 & 2 & 2 & 0 & 1 & 0 & 2 & 0 & 1\end{array}$

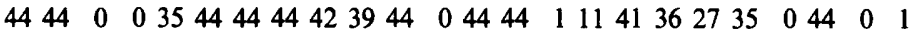

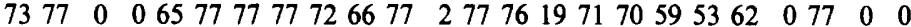
$\begin{array}{llllllllllllllllllllllll}5 & 5 & 0 & 0 & 5 & 5 & 5 & 5 & 4 & 5 & 5 & 0 & 5 & 5 & 2 & 4 & 5 & 5 & 4 & 5 & 0 & 5 & 0 & 0\end{array}$ $\begin{array}{llllllllllllllllllllllll}3 & 3 & 1 & 0 & 3 & 3 & 3 & 3 & 3 & 3 & 3 & 0 & 3 & 2 & 3 & 1 & 3 & 1 & 1 & 2 & 0 & 3 & 0 & 0\end{array}$ $\begin{array}{llllllllllllllllllllllll}6 & 6 & 0 & 0 & 5 & 6 & 6 & 6 & 6 & 0 & 6 & 0 & 6 & 6 & 2 & 6 & 6 & 6 & 5 & 6 & 1 & 6 & 0 & 0\end{array}$ $\begin{array}{llllllllllllllllllllllll}2 & 2 & 0 & 0 & 0 & 2 & 2 & 2 & 2 & 0 & 2 & 0 & 2 & 2 & 2 & 2 & 2 & 1 & 0 & 1 & 0 & 2 & 0 & 0\end{array}$ $\begin{array}{llllllllllllllllllllllll}4 & 4 & 0 & 0 & 4 & 4 & 4 & 4 & 2 & 1 & 4 & 0 & 4 & 4 & 2 & 4 & 0 & 4 & 4 & 4 & 0 & 4 & 0 & 0\end{array}$

$\begin{array}{llllllllllllllllllllllll}3 & 3 & 1 & 0 & 3 & 3 & 3 & 3 & 0 & 0 & 3 & 0 & 3 & 3 & 2 & 0 & 0 & 3 & 1 & 2 & 2 & 3 & 0 & 0\end{array}$

$\begin{array}{llllllllllllllllllllllll}2 & 2 & 0 & 0 & 2 & 2 & 2 & 2 & 2 & 2 & 2 & 0 & 2 & 2 & 0 & 0 & 2 & 2 & 0 & 0 & 2 & 2 & 0 & 0\end{array}$ $\begin{array}{llllllllllllllllllllllllll}11 & 11 & 0 & 0 & 11 & 11 & 11 & 11 & 11 & 11 & 11 & 0 & 11 & 7 & 0 & 1 & 11 & 7 & 0 & 1 & 10 & 11 & 10 & 11\end{array}$ $\begin{array}{llllllllllllllllllllllll}8 & 8 & 4 & 0 & 5 & 8 & 8 & 8 & 1 & 5 & 8 & 1 & 8 & 8 & 2 & 0 & 8 & 7 & 3 & 5 & 1 & 8 & 0 & 0\end{array}$ $\begin{array}{llllllllllllllllllllllll}5 & 5 & 5 & 0 & 5 & 5 & 5 & 5 & 3 & 5 & 5 & 0 & 5 & 5 & 4 & 0 & 5 & 5 & 4 & 5 & 2 & 5 & 0 & 0\end{array}$

$\begin{array}{llllllllllllllllllllllll}4 & 3 & 0 & 0 & 3 & 4 & 4 & 4 & 4 & 2 & 4 & 3 & 4 & 4 & 0 & 4 & 2 & 4 & 4 & 4 & 0 & 2 & 0 & 0\end{array}$ $\begin{array}{llllllllllllllllllllllll}2 & 1 & 0 & 0 & 0 & 1 & 1 & 2 & 0 & 0 & 2 & 2 & 2 & 2 & 0 & 2 & 2 & 2 & 2 & 2 & 0 & 2 & 0 & 0\end{array}$ $\begin{array}{llllllllllllllllllllllll}12 & 12 & 1 & 0 & 2 & 12 & 12 & 12 & 8 & 0 & 12 & 12 & 12 & 11 & 11 & 0 & 11 & 12 & 9 & 12 & 1 & 11 & 0 & 0\end{array}$ $\begin{array}{llllllllllllllllllllllll}7 & 7 & 0 & 0 & 0 & 7 & 7 & 7 & 4 & 1 & 7 & 7 & 7 & 7 & 7 & 2 & 7 & 2 & 6 & 7 & 0 & 7 & 0 & 0\end{array}$ $\begin{array}{llllllllllllllllllllllll}8 & 8 & 0 & 0 & 0 & 8 & 8 & 8 & 7 & 1 & 8 & 8 & 8 & 8 & 8 & 3 & 8 & 7 & 8 & 8 & 0 & 8 & 0 & 0\end{array}$ $\begin{array}{llllllllllllllllllllllll}2 & 2 & 0 & 0 & 0 & 2 & 2 & 2 & 0 & 0 & 2 & 2 & 2 & 2 & 2 & 0 & 2 & 0 & 1 & 2 & 0 & 0 & 0 & 0\end{array}$ $\begin{array}{llllllllllllllllllllllll}4 & 4 & 1 & 0 & 2 & 4 & 4 & 4 & 2 & 0 & 4 & 4 & 4 & 4 & 1 & 3 & 4 & 4 & 4 & 4 & 1 & 4 & 0 & 0\end{array}$ $\begin{array}{llllllllllllllllllllllll}0 & 3 & 3 & 0 & 3 & 3 & 3 & 3 & 0 & 1 & 3 & 3 & 3 & 0 & 2 & 0 & 3 & 3 & 1 & 2 & 0 & 3 & 0 & 0\end{array}$ $\begin{array}{llllllllllllllllllllllll}13 & 11 & 1 & 0 & 13 & 13 & 13 & 13 & 0 & 11 & 13 & 13 & 13 & 3 & 5 & 0 & 13 & 13 & 13 & 13 & 5 & 12 & 0 & 0\end{array}$ $\begin{array}{llllllllllllllllllllllll}2 & 2 & 0 & 0 & 1 & 2 & 2 & 2 & 0 & 1 & 2 & 0 & 2 & 0 & 0 & 0 & 2 & 1 & 2 & 2 & 1 & 2 & 0 & 0\end{array}$ $\begin{array}{llllllllllllllllllllllll}4 & 4 & 2 & 0 & 2 & 4 & 4 & 4 & 4 & 3 & 4 & 0 & 4 & 1 & 0 & 0 & 4 & 1 & 3 & 4 & 1 & 4 & 3 & 2\end{array}$

$\begin{array}{llllllllllllllllllllllll}9 & 9 & 1 & 0 & 9 & 9 & 9 & 9 & 7 & 8 & 9 & 0 & 9 & 8 & 0 & 1 & 9 & 0 & 4 & 4 & 0 & 9 & 8 & 0\end{array}$ $\begin{array}{llllllllllllllllllllllll}2 & 2 & 0 & 0 & 2 & 2 & 2 & 2 & 2 & 2 & 2 & 0 & 2 & 2 & 0 & 0 & 2 & 1 & 2 & 2 & 0 & 2 & 2 & 0\end{array}$ $\begin{array}{llllllllllllllllllllllll}10 & 10 & 2 & 0 & 2 & 10 & 10 & 10 & 10 & 4 & 10 & 4 & 10 & 0 & 3 & 0 & 10 & 8 & 4 & 8 & 6 & 10 & 0 & 0\end{array}$ $\begin{array}{llllllllllllllllllllllll}3 & 3 & 0 & 0 & 1 & 3 & 3 & 3 & 2 & 0 & 3 & 2 & 3 & 1 & 3 & 1 & 3 & 3 & 2 & 2 & 0 & 3 & 0 & 0\end{array}$ $\begin{array}{llllllllllllllllllllllll}3 & 3 & 0 & 0 & 1 & 3 & 3 & 3 & 0 & 0 & 0 & 0 & 3 & 0 & 0 & 0 & 2 & 2 & 2 & 2 & 0 & 3 & 0 & 2\end{array}$

$\begin{array}{llllllllllllllllllllllll}5 & 5 & 0 & 0 & 2 & 5 & 5 & 5 & 1 & 0 & 5 & 2 & 5 & 1 & 0 & 1 & 4 & 2 & 2 & 4 & 1 & 5 & 0 & 4\end{array}$ $\begin{array}{llllllllllllllllllllllll}2 & 2 & 1 & 0 & 2 & 2 & 2 & 2 & 2 & 1 & 1 & 0 & 2 & 2 & 0 & 0 & 2 & 2 & 2 & 2 & 0 & 2 & 0 & 0\end{array}$

$\begin{array}{llllllllllllllllllllllll}1 & 1 & 0 & 0 & 2 & 1 & 2 & 2 & 0 & 0 & 2 & 2 & 2 & 0 & 0 & 0 & 1 & 1 & 1 & 2 & 0 & 1 & 0 & 0\end{array}$ $\begin{array}{llllllllllllllllllllllll}6 & 7 & 0 & 7 & 6 & 7 & 7 & 7 & 3 & 2 & 7 & 7 & 7 & 7 & 7 & 1 & 7 & 3 & 0 & 3 & 2 & 1 & 0 & 0\end{array}$ $\begin{array}{llllllllllllllllllllllll}3 & 3 & 0 & 0 & 3 & 3 & 3 & 3 & 1 & 3 & 3 & 3 & 3 & 0 & 2 & 0 & 2 & 1 & 0 & 0 & 0 & 0 & 0 & 0\end{array}$ $\begin{array}{llllllllllllllllllllllll}5 & 1 & 0 & 0 & 0 & 6 & 5 & 6 & 0 & 0 & 2 & 3 & 6 & 6 & 6 & 2 & 5 & 4 & 0 & 0 & 3 & 6 & 0 & 0\end{array}$ 
Table 2 (continued)

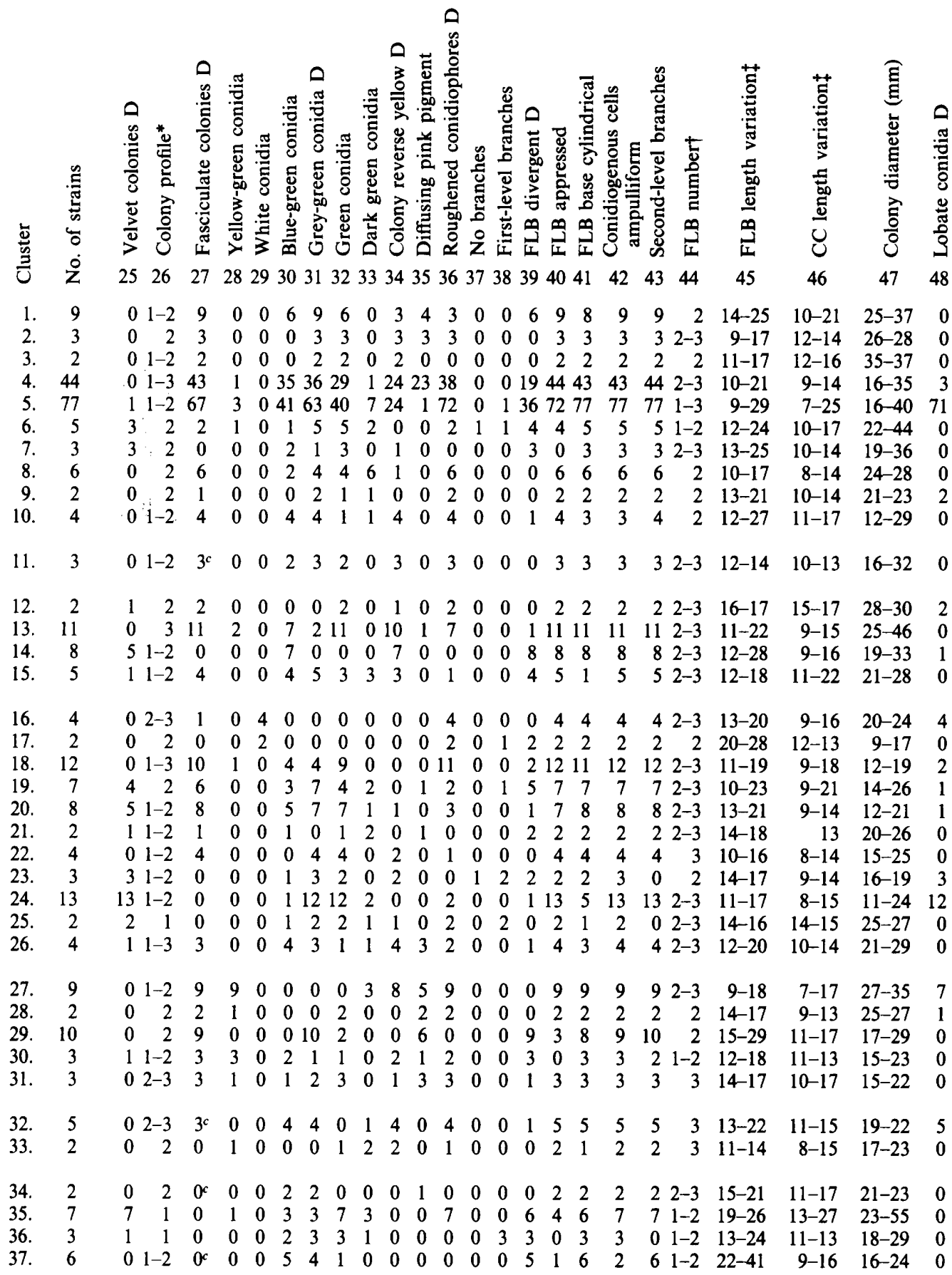

* Colony profile: 1 , flat and spreading; 2 , raised; 3 , high or domed.

† First-level branch number: 0, none; 1, 1-3 FLB; 2, 3-4 FLB; 3, >4 FLB.

$\ddagger$ First-level branch and conidiogenous cell length; coefficient of variation $=\mathrm{SD} /$ mean.

c Some or all strains produced coremia: $P$. granulatum II, $1 / 3$; coremial strains, $2 / 5 ; P$. clavigerum, $2 / 2$;

P. claviforme, $6 / 6$. 
Cluster

1. P. expansum

2. P. expansum

3. $P$. expansum

4. $P$. aurantiogriseum

5. $P$. solitum

6. P. aurantiogriseum

7. P. chrysogenum

8. P. echinulatum

9. P. olivinoviride

10. P. granulatum var. globosum

11. P. granulatum var. granulatum

12. P. hirsutum

13. P. hordei

14. P. chrysogenum

15. P. glandicola var. mononematosa

16. P. camembertii

17. Penicillium sp.

18. P. verrucosum

19. P. atramentosum

20. P. atramentosum

21. P. atramentosum

22. Penicillium sp.

23. $P$. citrinum

24. P. brevicompactum

25. P. raistrickii

26. P. aurantiogriseum var. neoechinulatum

27. P. viridicatum

28. $P$. viridicatum

29. P. griseofulvum

30. Penicillium sp.

31. P. hirsutum var. allii

32. Coremial strains

33. P. aurantiogriseum var. melanoconidium

34. P. clavigerum

35. P. roquefortii

36. P. corylophilum

37. P. claviforme
Table 2 (continued)
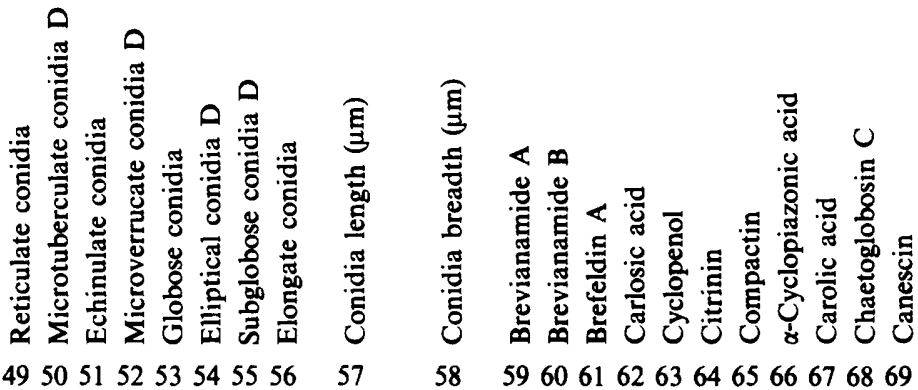

5960616263646566676869

$\begin{array}{lllllllllllllllllllllll}0 & 9 & 0 & 0 & 5 & 7 & 0 & 1 & 2.5-3.0 & 2.0 & -3.0 & 0 & 0 & 0 & 1 & 8 & 6 & 0 & 1 & 1 & 2 & 0\end{array}$ $\begin{array}{llllllllllllllllllllll}0 & 3 & 0 & 0 & 0 & 2 & 2 & 0 & 3 \cdot 0 & 2 \cdot 0-2 \cdot 5 & 0 & 0 & 0 & 0 & 2 & 0 & 1 & 0 & 0 & 0 & 0\end{array}$ $\begin{array}{llllllllllllllllllllll}0 & 0 & 0 & 2 & 0 & 2 & 0 & 0 & 2 \cdot 5-3 \cdot 0 & 2 \cdot 0-2 \cdot 5 & 1 & 0 & 0 & 0 & 2 & 0 & 0 & 0 & 0 & 0 & 0\end{array}$

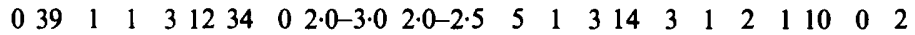

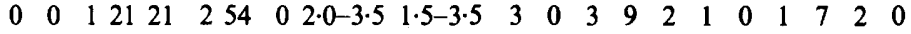
$\begin{array}{llllllllllllllllllllll}0 & 5 & 0 & 0 & 0 & 4 & 3 & 0 & 2.5-3.5 & 2.0-3.0 & 0 & 0 & 0 & 0 & 2 & 1 & 0 & 0 & 1 & 1 & 0\end{array}$ $\begin{array}{lllllllllllllllllllll}0 & 3 & 0 & 0 & 0 & 0 & 3 & 0 & 2 \cdot 5-3 \cdot 0 & 2 \cdot 0 & 0 & 0 & 0 & 0 & 0 & 0 & 0 & 0 & 0 & 0 & 0\end{array}$ $\begin{array}{llllllllllllllllllllll}0 & 0 & 6 & 0 & 3 & 0 & 3 & 0 & 3.0 & -3.5 & 3.0 & 0 & 0 & 1 & 0 & 0 & 0 & 2 & 0 & 0 & 0 & 3\end{array}$ $\begin{array}{lllllllllllllllllllllll}0 & 0 & 0 & 0 & 0 & 0 & 2 & 0 & 3 \cdot 0 & 2 \cdot 5-3 \cdot 0 & 0 & 0 & 1 & 0 & 0 & 1 & 0 & 0 & 0 & 0 & 0\end{array}$ $\begin{array}{llllllllllllllllllllll}0 & 4 & 0 & 0 & 4 & 0 & 0 & 0 & 2 \cdot 5 & 2 \cdot 0-2 \cdot 1 & 0 & 0 & 0 & 0 & 0 & 1 & 1 & 0 & 2 & 2 & 0\end{array}$ $\begin{array}{lllllllllllllllllllll}0 & 3 & 0 & 0 & 0 & 3 & 0 & 0 & 2 \cdot 5-3 \cdot 0 & 2 \cdot 0-2 \cdot 5 & 0 & 0 & 0 & 0 & 0 & 2 & 0 & 0 & 0 & 0 & 0\end{array}$ $\begin{array}{lllllllllllllllllllll}0 & 0 & 0 & 0 & 0 & 0 & 2 & 0 & 2.5 & 2.5 & 0 & 1 & 0 & 1 & 0 & 0 & 1 & 0 & 2 & 2 & 0\end{array}$ $\begin{array}{llllllllllllllllllllll}10 & 1 & 0 & 0 & 2 & 0 & 9 & 0 & 2 \cdot 5 & 2 \cdot 0 & -2 \cdot 5 & 1 & 0 & 0 & 6 & 7 & 8 & 0 & 0 & 6 & 1 & 0\end{array}$ $\begin{array}{lllllllllllllllllllll}0 & 7 & 0 & 0 & 2 & 1 & 6 & 0 & 2 \cdot 5-2 \cdot 7 & 2 \cdot 0-2 \cdot 5 & 1 & 0 & 0 & 0 & 0 & 0 & 0 & 0 & 0 & 0 & 0\end{array}$ $\begin{array}{llllllllllllllllllllll}0 & 5 & 0 & 0 & 0 & 0 & 5 & 0 & 2 \cdot 5-3 \cdot 0 & 2 \cdot 0-2 \cdot 4 & 0 & 0 & 0 & 0 & 0 & 0 & 0 & 0 & 0 & 0 & 0\end{array}$

$\begin{array}{lllllllllllllllllllll}0 & 0 & 0 & 0 & 0 & 2 & 4 & 0 & 2 \cdot 0-3 \cdot 7 & 2 \cdot 0-3 \cdot 7 & 0 & 0 & 0 & 0 & 0 & 0 & 0 & 0 & 0 & 0 & 0\end{array}$ $\begin{array}{llllllllllllllllllllll}0 & 1 & 0 & 1 & 1 & 0 & 1 & 0 & 2 \cdot 0 & 2 \cdot 0 & 0 & 0 & 0 & 0 & 0 & 2 & 0 & 0 & 0 & 0 & 0\end{array}$

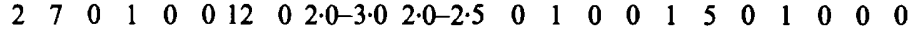
$\begin{array}{lllllllllllllllllllll}0 & 1 & 0 & 5 & 7 & 0 & 0 & 0 & 2 \cdot 0-3 \cdot 0 & 2 \cdot 0-2 \cdot 5 & 1 & 0 & 1 & 0 & 1 & 1 & 0 & 1 & 1 & 0 & 0\end{array}$ $\begin{array}{lllllllllllllllllllll}0 & 4 & 0 & 3 & 0 & 8 & 0 & 0 & 2 \cdot 5-3 \cdot 5 & 2 \cdot 0-2 \cdot 5 & 0 & 0 & 0 & 1 & 0 & 0 & 3 & 0 & 0 & 0 & 0\end{array}$ $\begin{array}{lllllllllllllllllllll}0 & 0 & 0 & 2 & 0 & 2 & 0 & 0 & 2 \cdot 7-2 \cdot 7 & 2 \cdot 5 & 0 & 0 & 0 & 0 & 0 & 0 & 0 & 0 & 0 & 0 & 0\end{array}$

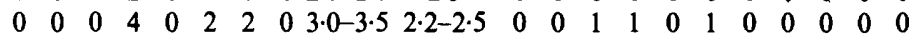
$\begin{array}{llllllllllllllllllllll}0 & 0 & 0 & 0 & 1 & 0 & 2 & 0 & 2 \cdot 0 & 1.5-1.8 & 0 & 0 & 0 & 0 & 0 & 3 & 0 & 0 & 0 & 0 & 0\end{array}$ $\begin{array}{lllllllllllllllllllll}1 & 0 & 0 & 0 & 1 & 1 & 12 & 0 & 2 \cdot 0-3 \cdot 0 & 2 \cdot 0-2 \cdot 5 & 3 & 2 & 0 & 0 & 0 & 1 & 1 & 1 & 0 & 1 & 0\end{array}$ $\begin{array}{llllllllllllllllllllll}0 & 0 & 0 & 2 & 0 & 0 & 2 & 0 & 2 \cdot 0 & 1 \cdot 5-2 \cdot 0 & 1 & 0 & 0 & 0 & 0 & 0 & 0 & 0 & 0 & 0 & 0\end{array}$ $\begin{array}{lllllllllllllllllllll}0 & 0 & 4 & 0 & 2 & 0 & 2 & 0 & 2.5-3.0 & 2 \cdot 5-3.0 & 0 & 0 & 0 & 3 & 0 & 0 & 0 & 0 & 0 & 0 & 0\end{array}$

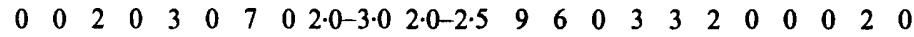
$\begin{array}{lllllllllllllllllllllll}0 & 1 & 0 & 0 & 1 & 0 & 1 & 0 & 2 \cdot 5 & 2 \cdot 0-2 \cdot 5 & 0 & 0 & 0 & 0 & 0 & 0 & 0 & 0 & 0 & 0 & 0\end{array}$ $\begin{array}{lllllllllllllllllllll}0 & 10 & 0 & 0 & 0 & 8 & 6 & 0 & 2 \cdot 0-3 \cdot 0 & 2 \cdot 0-2 \cdot 5 & 0 & 0 & 0 & 2 & 3 & 0 & 0 & 1 & 0 & 1 & 0\end{array}$ $\begin{array}{llllllllllllllllllllll}0 & 3 & 0 & 0 & 0 & 0 & 3 & 0 & 2 \cdot 5 & 2 \cdot 0-2 \cdot 5 & 0 & 0 & 0 & 1 & 0 & 0 & 0 & 0 & 0 & 0 & 1\end{array}$ $\begin{array}{llllllllllllllllllllll}0 & 2 & 0 & 1 & 0 & 0 & 3 & 0 & 2.5-3.0 & 2.5 & 0 & 0 & 0 & 1 & 0 & 0 & 1 & 0 & 0 & 0 & 0\end{array}$ $\begin{array}{lllllllllllllllllllll}0 & 0 & 0 & 0 & 0 & 0 & 5 & 0 & 2 \cdot 5-3 \cdot 0 & 2 \cdot 5-2 \cdot 7 & 0 & 0 & 1 & 2 & 0 & 1 & 1 & 1 & 0 & 0 & 1\end{array}$ $\begin{array}{lllllllllllllllllllll}0 & 2 & 0 & 0 & 0 & 2 & 0 & 0 & 2 \cdot 7-3 \cdot 2 & 2 \cdot 5 & 0 & 0 & 0 & 1 & 0 & 0 & 1 & 0 & 0 & 0 & 1\end{array}$

$\begin{array}{llllllllllllllllllllll}0 & 0 & 0 & 2 & 0 & 2 & 0 & 0 & 2.5-3.0 & 2 \cdot 0-2.5 & 2 & 0 & 0 & 0 & 1 & 1 & 0 & 0 & 0 & 0 & 0\end{array}$ $\begin{array}{lllllllllllllllllllll}1 & 6 & 0 & 0 & 6 & 0 & 1 & 0 & 3 \cdot 0-4 \cdot 0 & 2 \cdot 5-3 \cdot 5 & 0 & 0 & 0 & 0 & 0 & 0 & 1 & 0 & 0 & 0 & 0\end{array}$ $\begin{array}{lllllllllllllllllllll}0 & 1 & 0 & 2 & 0 & 0 & 3 & 0 & 2 \cdot 0-3.0 & 2 \cdot 0-2.5 & 0 & 0 & 0 & 0 & 0 & 0 & 0 & 0 & 0 & 0 & 0\end{array}$ $\begin{array}{llllllllllllllllllllll}0 & 5 & 0 & 1 & 0 & 6 & 0 & 0 & 3.0 & 2.5 & 0 & 0 & 0 & 0 & 0 & 0 & 0 & 0 & 0 & 0 & 0\end{array}$ 
Table 2 (continued)

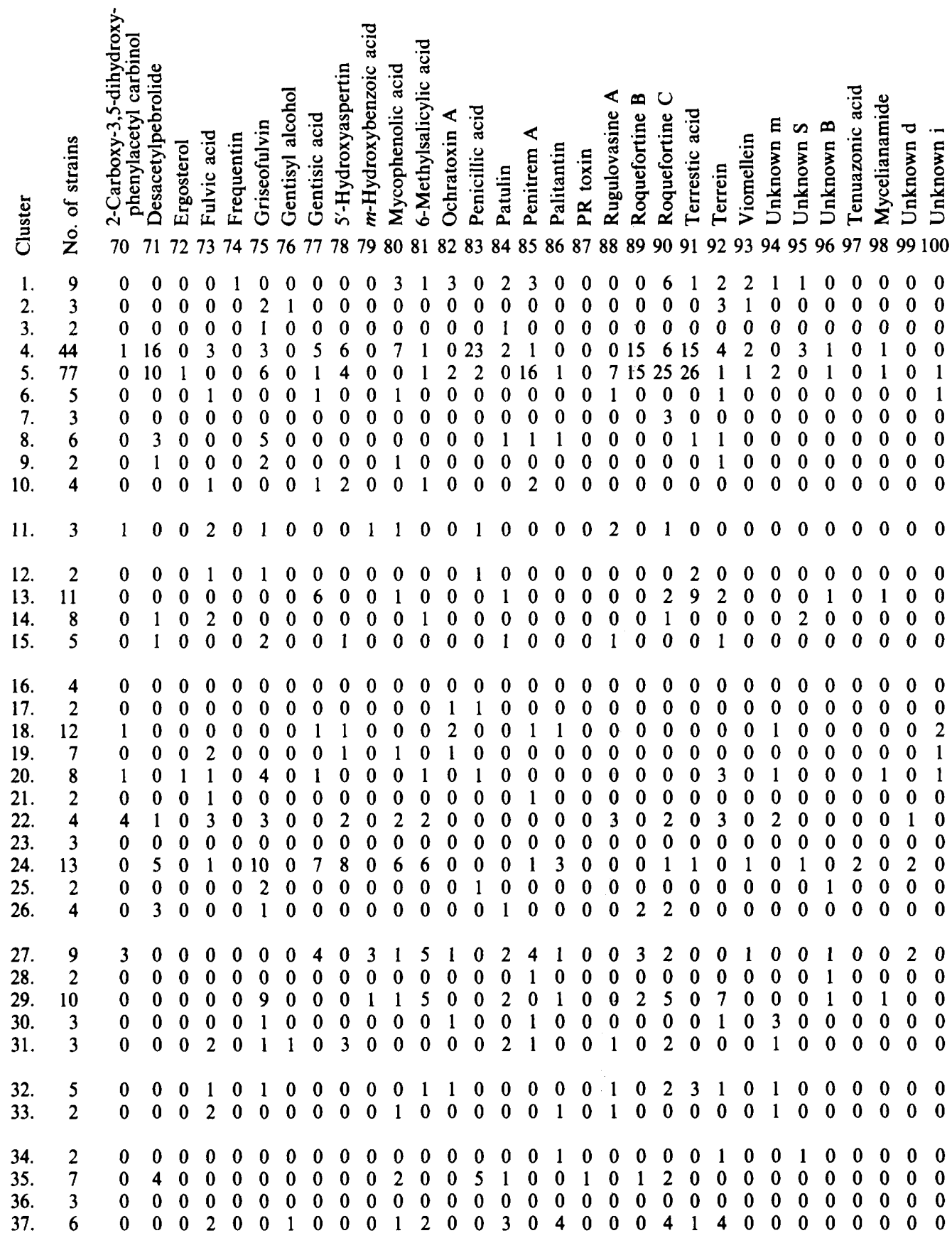




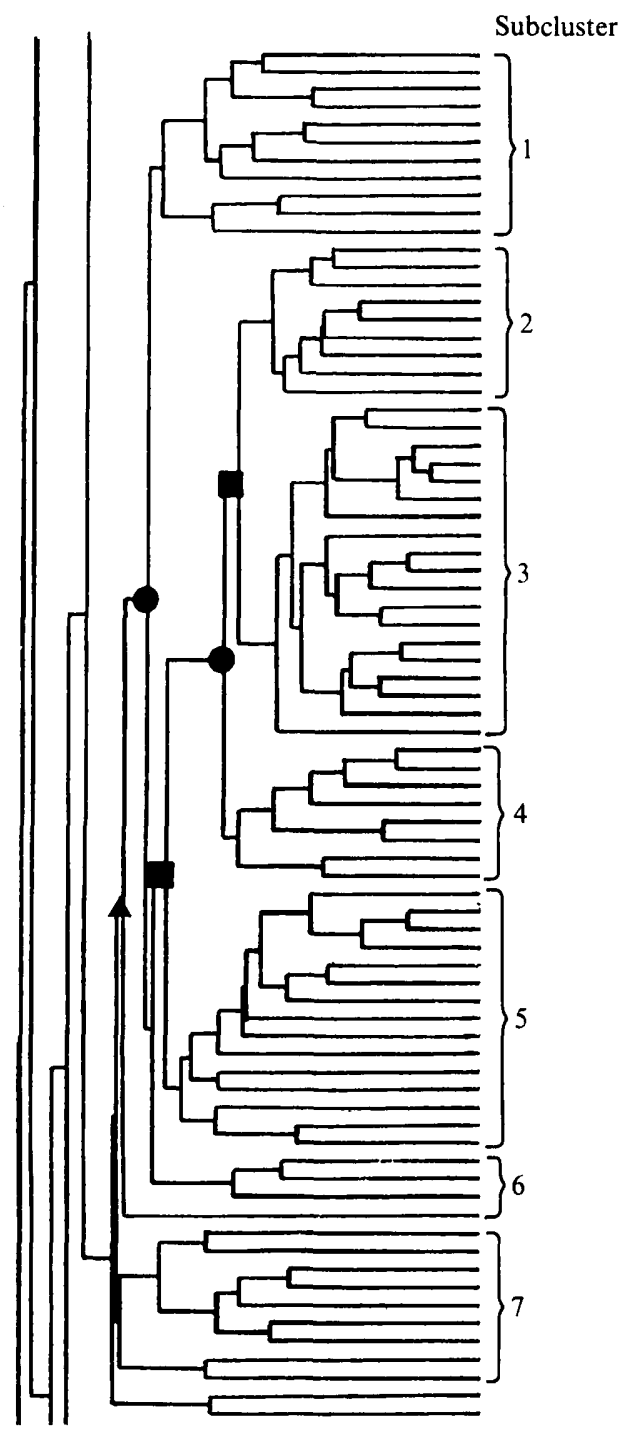

Fig. 2. Subclusters in cluster 5. $\Delta$, Branch point where $P<0.90$ that overlap is less than rectangular; 0 , branch point where $P>0.95$ that overlap is less than rectangular; $\boldsymbol{D}$, branch point where $P>0.99$ that overlap is less than rectangular.

in preservation for many years, and certainly a number of the names as received on the cultures may be of doubtful validity. The cluster was recovered as seven smaller clusters in the Gower's coefficient dendrogram (Fig. 2) and most of these could be recognized in the Pattern dendrogram. There were few distinguishing characters between these smaller clusters (Table 3). This area of the genus has been reported by several authors to consist of a number of species, some of which have been considered synonymous with $P$. aurantiogriseum, including P. commune, $P$. solitum, P. palitans and P. crustosum (see Samson et al., 1976; Pitt, 1980; Frisvad, 1985; Cruickshank \& Pitt, 1987). In this study the central part of the cluster appeared distinct, with properties gradually varying in subgroups away from this. Overlap statistics suggested that some subgroups were distinct, particularly within the arm containing subgroups 2-5 (see Fig. 2). However, the Euclidean distances, derived from the similarity values, for both inter- and intragroup distances were all very similar (Table 4). A principal coordinates analysis of these strains did separate the central region of cluster 5 but since only $28 \%$ of the variation was explained by 
Table 3. Most useful tests available for differentiating between subclusters of cluster 5 (P. solitum)

,$+>85 \%$ positive;,$-<25 \%$ positive; $v, 25-85 \%$ positive.

\begin{tabular}{|c|c|c|c|c|c|c|c|}
\hline & \multicolumn{7}{|c|}{ Subclusters } \\
\hline & 1 & 2 & 3 & 4 & 5 & 6 & 7 \\
\hline Production of penitrem $\mathrm{A}$ & - & - & - & $\mathbf{v}$ & $\mathrm{v}$ & - & - \\
\hline Growth on tannic acid & + & + & + & + & + & - & $\mathbf{v}$ \\
\hline DNA hydrolysis & - & $\mathrm{v}$ & $\mathbf{v}$ & + & + & + & + \\
\hline RNA hydrolysis & - & + & + & $\mathrm{v}$ & + & $\mathbf{v}$ & + \\
\hline Conidia blue-green & + & $\mathrm{v}$ & $\mathbf{v}$ & - & $\mathrm{v}$ & + & $\mathrm{v}$ \\
\hline FLB divergent & - & + & $\mathbf{v}$ & $\mathbf{v}$ & - & + & + \\
\hline Conidia globose & - & - & - & $\mathrm{v}$ & $\mathbf{v}$ & + & - \\
\hline Conidia subglobose & + & + & + & $\mathrm{v}$ & $\mathrm{v}$ & - & + \\
\hline Colony diameter $(\mathrm{mm})$ & $24-32$ & 24-34 & $27-40$ & $25-38$ & $16-28$ & $21-29$ & $22-34$ \\
\hline
\end{tabular}

Table 4. Inter- and intra-group Euclidean distances for subgroups of cluster 5 ( $P$. solitum)

$\begin{array}{cclllllll} & \begin{array}{c}\text { Sub- } \\ \text { cluster }\end{array} & & & & & & & \\ & 1 & 0 & & & & & & \\ & 2 & 1.67 & 0 & & & & & \\ & 3 & 2.18 & 1.75 & 0 & & & & \\ & 4 & 2.24 & 1.92 & 1.63 & 0 & & & \\ & 5 & 1.71 & 1.58 & 2.25 & 1.76 & 0 & & \\ & 6 & 2.45 & 2.16 & 2.77 & 2.25 & 2.13 & 0 & \\ & 7 & 2 \cdot 21 & 1.55 & 2.25 & 2.33 & 2.04 & 2.28 & 0 \\ \text { Mean intra-group distances } & & 1 & 2 & 3 & 4 & 5 & 6 & 7 \\ & & 2.23 & 1.69 & 1.65 & 2.04 & 1.97 & 2.44 & 1.98\end{array}$

the first three dimensions, this separation was at best slight. It therefore seems likely that the cluster represents a large continuum, showing almost random variation in most properties. The central region of the cluster corresponds well with descriptions of $P$. crustosum (e.g. Thom, 1930; Raper \& Thom, 1949; Pitt, 1980), and could be distinguished from the rest of the strains by secondary metabolite production, particularly terrestric acid and roquefortine $\mathrm{C}$, but the overall arrangement of the cluster suggested that this was only at best a variety of the overall taxon.

The number of older ex-type strains within this cluster makes assigning a name difficult. The oldest ex-type strain included is $P$. griseobrunneum. However, this name has previously been considered a synonym of $P$. brevicompactum (Samson et al., 1976; Pitt, 1980) and there may be some doubt about the authenticity of the line included here. Similarly, the next oldest name included in the group, $P$. biforme, has been considered a synonym of $P$. verrucosum var. cyclopium by Samson $e$ al al (1976), a synonym of $P$. camembertii by Pitt $(1980,1986)$, and has been included in $P$. commune in later studies (e.g. Polonelli et al., 1987). In the light of this, and the fact that the line used in this study showed both green- and white-spored sectors, it would be unwise to resurrect this old name. The ex-type cultures of $P$. solitum, $P$. palitans and $P$. majusculum were all present in this cluster. $P$. palitans has been considered a synonym of $P$. viridicatum by Pitt (1986), although this author later considered $P$. palitans as a synonym of $P$. commune (Cruickshank \& Pitt, 1987). P. majusculum was represented by two different lines of the ex-type culture in this study which were recovered in different places, so the authenticity of this strain must be in doubt. The best name, as represented by an ex-type strain, which can be applied to this taxon is $P$. solitum. The central area of the cluster could be distinguished as a variety, and the general description and metabolite production profile match fairly well with $P$. crustosum (Frisvad, 1986; Pitt, 1980, 1986). The following new combination is made $\boldsymbol{P}$. solitum var. crustosum (Thom) comb. nov. [basionym: P. crustosum Thom, Penicillia: 399, 1930]. Pitt (1986) designated IMI 91917 as the lectotype, and as herbarium material of this culture is still extant this specimen is acceptable as the nomenclatural type of the name. 
Linked above cluster 6 were four single isolates (single-member clusters 2-5; IMI 285531, 192904, 39819 and 89312). IMI 285531 was received as $P$. camembertii III, a name ascribed to certain strains of $P$. palitans (Frisvad, 1986), a taxon recovered in cluster 5; this strain may be an atypical member of this group. The other three strains were all ex-type cultures. The strains IMI 192904 and 39819 were the only isolates of $P$. psittacinum and $P$. lanosoviride included in the study and so little can be concluded from their position. The strain IMI 89312 is of particular interest; it was originally described as a white-spored mutant of $P$. cyclopium (i.e. $P$. aurantiogriseum) although during most of this study the line consistently produced fawn conidia. However, both white and blue-green sectors have been produced by this strain so it appears to be naturally unstable.

Cluster 6 consisted of five strains. There were few distinguishing characters between these and $P$. aurantiogriseum other than colony texture and that these strains have only slightly roughened or smooth conidiophores. The ex-type culture of $P$. puberulum was present in this cluster and strains accepted in $P$. puberulum by Pitt (1980) are typical of the cluster. The ex-type strain was one of the two cases of duplicate cultures not being recovered together, the other line clustering in $P$. aurantiogriseum. Cruickshank \& Pitt (1987) have considered $P$. puberulum to be a synonym of $P$. aurantiogriseum and this view is supported here.

Cluster 7 consisted of three strains including the ex-type isolate of $P$. chrysogenum, although this is an old culture that is now in a poor condition and shows few distinctive morphological features. This cluster moved close to other more recent isolates identified as $P$. chrysogenum (cluster 14) with the Pattern coefficient and so is considered to represent older and/or deteriorated representatives of this species.

Cluster 8 consisted of six strains including the ex-type strain of $P$. echinulatum. Echinulate conidia are rare amongst the fasciculate penicillia and so this was a useful diagnostic character for P. echinulatum which conformed to existing concepts (see Samson et al., 1976; Pitt, 1980).

Cluster 9 consisted of two unusual strains which were in poor condition, one of which was received as the ex-type strain of $P$. olivinoviride. In the absence of any other material this cluster was tentatively named as $P$. olivinoviride.

Three strains (IMI 285511, 293204 and 59286) were recovered on a separate arm of the dendrogram directly below clusters 8 and 9 . These strains also appeared as a weak group in the Pattern dendrogram. Two of them (IMI 285511 and 293204) were received as new chemotype variants of the two species $P$. hirsutum and $P$. roquefortii. The third was received as $P$. granulatum. The strains shared few common properties and so any decision would require the study of further isolates. Below this set of three were two further strains (IMI 285526 and 298085 ) which were partly attached to cluster 10 . These were both phenotypically unusual isolates, which were recovered separately in other parts of the Pattern dendrogram and so their identity remains unclear.

Clusters 10 and 11 consisted of two sets of three strains, with the ex-neotype of $P$. granulatum being recovered in cluster 11 . The two clusters were recovered separately in different areas of the Pattern dendrogram. Both groups showed many common properties but differed in conidial shape and the production of base from creatine, other differences being primarily a matter of degree only. The base from creatine reaction has been considered a species-specific character by Frisvad; however, he reported isolates of $P$. granulatum as being both creatine-positive and -negative (Frisvad, 1985, 1986). Pitt (1986) reported the conidia of $P$. granulatum as 'ellipsoidal, less commonly subspheroidal'. These two groups seem to represent varieties of the same species, cluster 11 being $P$. granulatum var. granulatum and cluster 10 being a creatine-positive $P$. granulatum variety with globose conidia. This new variety is named here as $\boldsymbol{P}$. granulatum var. globosum var. nov. [Diagnosis: ab varietate granulato differt quod habet conidia globosa et id quod anglicebase from creatin redditur producit. Holotypus: USA, Wisconsin, isolatus ex solo sylvae, 1983. S. E. Gochenaur (IMI 299049).]

Seifert \& Samson (1986) reexamined early herbarium specimens and considered dried material described in the genus Coremium as equivalent to a number of strongly coremial Penicillium species. As the coremium species epithets are older, they should have priority over the existing Penicillium species, thus renaming $P$. granulatum as $P$. glandicola (Seifert \& Samson, 
1986). However, the earlier material from the genus Coremium is available only as herbarium specimens and so a detailed objective comparison can not be made with the living cultures studied here. In our view it is unwise to resurrect old names in this genus, for which no ex-type cultures are extant, as the full range of parameters used in species separations in the genus cannot be ascertained; thus some doubt must always remain (in the absence of further studies such as DNA homologies) as to whether the specimens are truly conspecific with the available cultures.

Clusters 12 and 13 consisted of 13 strains recovered in both dendrograms as a distinct arm of two strains (cluster 12) and a core group of 11 (cluster 13). The larger group contained the ex-neotype of $P$. hirsutum, which is synonymous with $P$. corymbiferum (Pitt, 1980). The cluster also contained the ex-type strain of $P$. hordei, which has been considered a synonym of $P$. hirsutum (Pitt, 1980), although the species is now regarded as distinct by Frisvad (1985) and Cruickshank \& Pitt (1987). Unusually for fasciculate penicillia, these isolates were able to hydrolyse cellulose and produced a yellow pigment from CZNH4 agar. The two strains in cluster 12 differed in conidial ornamentation and in not producing citrinin or any yellow pigmentation. It has been suggested that these two latter properties may be linked (e.g. Smith, 1949). P. hordei was differentiated from $P$. hirsutum partly on the basis of the rough-walled conidia of the former (Samson et al., 1976), a property seen in this study, where strains in cluster 13 have distinctly reticulate conidia in comparison to the finely lobate conidia of strains in cluster 12 . It therefore seems likely that this small cluster represents $P$. hirsutum, although further work is necessary to establish whether there is in fact a gradation between this and $P$. hordei. At the base of cluster 13 was a single-member cluster (SM6) which contained strain IMI 19759, received as $P$. cyclopium; this remained ungrouped in the Pattern dendrogram.

Cluster 14 consisted of eight strains received as $P$. chrysogenum or related species and the ex-type culture of the older name $P$. griseoroseum. Samson et al. (1977) commented on the relationship between $P$. griseoroseum and $P$. chrysogenum, regarding the former as a synonym of the latter, a view supported in other recent work (Cruickshank \& Pitt, 1987). This cluster is very similar to cluster 7 which contained the ex-type culture of $P$. chrysogenum, although strains in cluster 14 differed in producing base from lactic acid, having blue-green conidia and some appressed branches. Strains from cluster 14 also failed to produce roquefortine $C$ consistently. In this case, where both clusters contained ex-type strains, they could be considered as two different species. However, the two clusters showed few different characters, most discrepancies probably being due to the vigour of the isolates. This is not a complete explanation as the two clusters were recovered close together but separate in the Pattern dendrogram. The characteristics of both clusters correspond well with the accepted concept of $P$. chrysogenum (e.g. Samson et al., 1977; Pitt, 1980). Our line of the ex-type strain of $P$. notatum, considered a synonym of $P$. chrysogenum, was very deteriorated and was not recovered in this cluster. Given these factors, possible deterioration of some of the older ex-type cultures, and the importance of the name $P$. chrysogenum, it is prudent to consider both clusters as variants of $P$. chrysogenum until further isolates can be studied.

Two strains were recovered loosely linked at the top of cluster 15 (IMI 75832 and 321507). Strain 75832, originally received as the ex-type of $P$. griseofulvum, was found to be contaminated at the end of the study and so must be discounted. Strain 321507 was one of two representatives of $\boldsymbol{P}$. chrysogenum var. dipodomysis (Frisvad et al., 1987). The other strain of this species (IMI 321508) was recovered loosely linked at the base of cluster 15 . These two isolates moved considerably in the Pattern dendrogram and were recovered in a number of different positions in other representations (unpublished). It is possible that these strains are true 'wanderers' (Sackin, 1985) or that this species is intermediate between several others where minor differences in characters may have significant taxonomic effect. More strains of $P$. chrysogenum var. dipodomysis are needed before any firm taxonomic conclusions can be drawn.

Cluster 15 consisted of five strains, four of $P$. glandicola var. mononematosa, including the ex-type culture and one strain of $P$. flavogenum. This cluster represents $P$. glandicola var. mononematosa but the relationship with $P$. flavogenum is unclear. This isolate appeared at the base of the group as a possible outlier and so may be distinct. However, there are insufficient 
strains to make a firm decision. At the base of clusters 14 and 15 there were two pairs of ungrouped strains. The upper pair consisted of $P$. chrysogenum var. dipodomysis, discussed above, and a further isolate labelled 'viridicatum VII' (IMI 269930). This strain was considered distinct by J. C. Frisvad (personal communication), but to date no similar strains have been isolated and so its position cannot be properly determined. The second pair of strains were the ex-type culture of $P$. nigricans (IMI 39767), a species considered in synonymy with $P$. janczewskii (Pitt, 1980), and one strain received as $P$. cyclopium (IMI 297544). $P$. nigricans is not terverticillate and was included as an example of a different, distantly related species. However, $P$. nigricans is assigned to the section Divaricatum (Pitt, 1980), species of which are characterized by an irregular branching pattern that could be considered terverticillate. Strain 297544 , received as $P$. cyclopium, does not belong to that taxon and appears to be a deteriorated isolate of a different species.

Clusters 16 and 17 consisted of six strains split into one group of four and a smaller group of two in both dendrograms. Five of the six strains were received as $P$. camembertii or synonyms, including the ex-type culture. The two clusters were distinguished by the production of citrinin, their conidial ornamentation and several negative physiological characters for cluster 17 . Citrinin production has not been reported in P. camembertii before. Cluster 16 was citrininnegative and contained strains recently isolated from cheese, whereas the apparent citrininpositive cluster 17 consisted of two older ex-type strains. One possible explanation is that cluster 16 represents the true cheese $P$. camembertii strains, whereas the older ex-type strains in cluster 17 represent a different taxon. Included in cluster 16 was one strain (IMI 287746) which was isolated as a white-spored sector from a strain recovered in cluster 5. This white variant remained unstable throughout the work, often reverting to green conidia. $P$. camembertii has been suggested as a domesticated variant of the green-spored $P$. commune (Polonelli et al., 1987; El-Banna et al., 1987), and the placement of this strain in cluster 16 gives some support to that theory.

Cluster 18 consisted of 12 strains but no ex-type cultures. Six of these strains have previously been identified as $P$. verrucosum and the overall morphological description of this cluster matches the species concept of Pitt (1980). The ex-type culture of $P$. verrucosum was recovered in the adjacent cluster 19 in this dendrogram, but was found in this cluster with the Pattern dendrogram. It is likely that the ex-type culture is either deteriorated or in a generally poor condition.

Clusters 19 and 20 consisted of 15 strains split into two clusters at $69 \%$ similarity; they differed primarily in conidial shape. These two clusters were recovered as one single cluster in the Pattern dendrogram, along with cluster 21 . The only ex-type culture recovered in these clusters was that of $P$. verrucosum (see above). Strain IMI 192503 was described as indistinguishable from the lectotype of $P$. atramentosum by Pitt (1980) and his general description of this species agrees well with the morphological characters recorded here. The ex-lectotype culture of $P$. atramentosum was included in this study (IMI 39752) but proved to be very deteriorated and was loosely linked near the base of the dendrogram. A fresh line of this culture examined subsequently agreed with all the typical properties of the clusters (Bridge et al., 1989). Cluster 19 therefore represents $P$. atramentosum, with cluster 20 being an elliptical-spored variant.

Cluster 21 consisted of only two strains, both received as $P$. atramentosum, which had elliptical conidia and failed to hydrolyse starch. These two characters were the only clear-cut differences between this cluster and clusters 19 and 20 ; the group can thus be considered a starch-negative variant of $P$. atramentosum.

Cluster 22 consisted of four strains received as two new varieties of $P$. glandicola and $P$. coprophilum. All produced 2-carboxy-3,5-dihydroxyphenylacetyl carbinol, a metabolite not commonly found in this group of penicillia. This cluster may represent two taxa, as the morphology of the strains was quite varied; however, more isolates are needed to make a firm decision.

Three strains (IMI 285510, 296073 and 134650) appeared below cluster 22 as single-member clusters (SM7, 8 and 9), loosely linked to clusters $1-22$. These are all apparently unrelated strains 
and they included one line of the ex-type culture of $P$. majusculum (IMI 134650). Another line of this culture (IMI 321523), received through a different route had possibly become replaced and was recovered in cluster 5 . The three strains appear to be three distinct isolates but some further study is needed to clarify their position.

Cluster 23 consisted of three strains including the ex-type isolate of $P$. citrinum and one isolate obviously replaced by $P$. citrinum. This species was included in the study as an example of a distantly related species. The placement of the species here rather than as a distinct outlier may be due to some of the typical diagnostic properties of this organism, which include the production of citrinin and other yellow pigments, like many terverticillate penicillia, and the general gross colony morphology being similar to those of $P$. brevicompactum.

Cluster 24 consisted of 13 strains, including the ex-neotype cultures of $P$. brevicompactum and $P$. olsonii, and the ex-type culture of $P$. stoloniferum. $P$. stoloniferum was regarded as a synonym of $P$. brevicompactum by Pitt (1980), and the latter is the oldest name for this group. P. olsonii is generally regarded as distinct (Pitt, 1980; R. A. Samson, personal communication), although in this study there appeared to be no features that could distinguish it from $P$. brevicompactum.

Cluster 25 consisted of two strains, including the ex-type of $P$. raistrickii. This species was included for comparison and is only distantly related to the terverticillate penicillia.

Loosely linked at the top of cluster 26 was a single-member cluster (SM10) received as $P$. corymbiferum (IMI 68414). This strain was morphologically typical of $P$. hirsutum, with which $P$. corymbiferum is synonymous (Pitt, 1980). However, this strain was atypical both physiologically and biochemically and does not appear to group with any accepted species.

Cluster 26 formed at below $70 \%$ similarity and contained three strains received as $P$. aurantiogriseum var. neoechinulatum and one strain described as atypical $P$. hirsutum. This group differed from $P$. echinulatum primarily by having yellow mycelium and being creatinenegative.

Below cluster 26 were three loosely linked strains: two of these (IMI 92209ii and 297546) were linked at $70 \%$ similarity and were representatives of two species, $P$. citreoviride and $P$. raciborski, which are both unrelated to the terverticillate penicillia and unrelated to each other. The third strain (IMI 293178) was received as $P$. camembertii but had presumably been originally misidentified as it produced green, echinulate conidia.

Cluster 27 contained nine strains including the ex-type strain of $P$. viridicatum. The properties recorded agreed well with the concept of this species given by Pitt (1980).

Linked at the base of cluster 27 was the small cluster 28 , consisting of two strains, which were very similar to $P$. viridicatum and joined that cluster at $67 \%$ similarity. However, these two strains were found loosely linked at the base of $P$. aurantiogriseum in the Pattern dendrogram. $P$. viridicatum and $P$. aurantiogriseum appear well-separated on the dendrograms but they showed significant overlap (see later). These two strains represent a variant of $P$. viridicatum intermediate between the two taxa.

Below clusters 27 and 28 were three loosely linked strains; one (IMI 291201) was received as the ex-type of $P$. ochraceum var. macrosporum. These remained loosely linked in the same position in the Pattern dendrogram and may represent atypical variants of $P$. viridicatum.

Cluster 29 contained ten strains, identified as $P$. griseofulvum or currently accepted synonyms. The ex-type culture of this species was not present in the cluster as discussed above (under cluster 15). However, subsequent testing placed a fresh ex-type line in this cluster.

Below cluster 29 were four loosely linked strains and cluster 30 . This cluster consisted of three $P$. viridicatum-like strains and was recovered in a similar position in the Pattern dendrogram. Cluster 30 represents a group of three different strains, all of which were in poor condition.

Cluster 31 consisted of two strains considered a new taxon by J. C. Frisvad (personal communication), P. hirsutum var. allii and one additional strain. They were loosely linked at $59 \%$ similarity but appear distinct.

Cluster 32 consisted of five loosely linked strains that were mainly received as further unpublished varieties of $P$. hirsutum. Cluster 31 and 32 were recovered together in the Pattern dendrogram and strains in both clusters formed coremia. Cluster 32 may be merely a variant of cluster 31 . 
Cluster 33 consisted of two strains recognized as a new variety, $P$. aurantiogriseum var. melanoconidium (J. C. Frisvad, personal communication). Loosely linked to this cluster was a single coremial strain which had been misidentified as $P$. claviforme. Clusters 31-33 may represent a single new taxon or atypical variants of an existing one.

Cluster 34 consisted of two strains (including the ex-type) of $P$. clavigerum. Above this cluster were two loosely linked strains: both were very poor deteriorated cultures.

Cluster 35 consisted of seven strains of $P$. roquefortii, including the ex-type strain. This cluster was distinct in a number of features including growth on acetic acid agar and colony size.

Cluster 36 consisted of three strains, including the ex-type, of $P$. corylophilum. This was a distinct group in both dendrograms and is not closely related to the fasciculate penicillia.

Below cluster 36 were two sets of loosely linked strains. These included the only three authentic lines of $P$. italicum included in the study; the remainder were all either representatives of distantly related species or poorly growing isolates in a deteriorated condition.

Cluster 37 consisted of six strains of the strongly coremial $P$. claviforme. This taxon, included for comparison, was distinct on both dendrograms; it is not closely related to the fasciculate species. As with $P$. granulatum, an earlier species epithet is available from Coremium vulpinum (Seifert \& Samson, 1986). Again, we consider it unwise to resurrect this name in the absence of extant ex-type cultures.

At the base of the dendrogram was a single-member cluster (SM11), containing a strain of $P$. funiculosum. this was included as an example of a different section of the genus, subgenus Biverticillium, and so was expected to be distinct from other strains.

\section{Vigour}

The vigour values for the strains used in this study varied from 0.198 to 0.419 . However, the mean vigour values for the clusters described ranged only between 0.26 and 0.35 . This is a narrow range and indicates that groups formed from deteriorated cultures in this study are not necessarily less vigorous for the characters used. An example of this is cluster 6 , considered a deteriorated variant of $P$. aurantiogriseum. The mean vigour for cluster $6(0.325)$ is actually higher than that for $P$. aurantiogriseum $(0 \cdot 317)$. $P$. chrysogenum was recovered in two wellseparated clusters in the Gower dendrogram, clusters 7 and 14, and these clusters moved closer together in the Pattern dendrogram. Vigour differences were not apparently responsible for this as the mean vigour of $P$. chrysogenum $\mathrm{I}$, cluster 7 , was 0.281 and the mean vigour of $P$. chrysogenum II, cluster 14 , was $0 \cdot 262$. In $P$. expansum, clusters 1 and 3 , where cluster 3 contained freshly isolated strains, the vigour for this cluster was slightly lower than for the main group (cluster 1). One possible reason for this is that when deteriorated strains start to lose characteristic properties, they may express others: for example, they may stop producing a particular secondary metabolite but instead accumulate a precursor which is seen as a diffusing pigment. This has been suggested as a possibility with some red pigments that may be due to an overproduction of melanin following deregulation of metabolite pathways (see Anné, 1986).

\section{CONCLUSIONS}

The fasciculate penicillia have many common properties and overall they appear very similar. Serological studies have shown considerable similarity between these species, most antisera being unable to separate them (Polonelli et al., 1986, 1987). Patterns of extracellular enzymes have shown distinct separations at only the subgenus and section level (Bridge \& Hawksworth, 1984) and protoplast fusion studies have resulted in cross-specific heterokaryons (Anné \& Peberdy, 1981; Anné, 1986). The mol \% G + C ratios for penicillia are all very similar (e.g. Storck \& Alexopoulos, 1970), and first-derivative melting curve data from DNA of representative strains of the present study also indicate considerable similarity, particularly between fasciculate strains (Paterson et al., 1990). To date no DNA hybridization data have been published for Penicillium. Electrophoretic mobilities of pectinase and amylase enzymes have been proposed as species-specific by Cruickshank \& Pitt (1987), who used 181 strains from 38 species or synonyms. In our experience numerical taxonomic studies on pectinase and amylase isozymes from some of the cultures included in this study have shown there may be 
considerable heterogeneity in isozyme patterns in many species (accompanying paper: Paterson et al., 1989). This study has shown that properties may vary considerably within some species as was seen in $P$. solitum, although the species remained distinct on other grounds. Interestingly, preliminary results suggest that there is very little difference in mitochondrial DNA restriction fragment length polymorphisms (RFLPs) from strains with diverse names and histories referred to as $P$. solitum here (J. Croft, unpublished results), indicating a common phylogeny (Croft, 1987).

The present results raise a number of interesting points. Firstly, the number and reproducibility of secondary metabolites seen in some of the strains studied here were in general lower than those reported by some workers (e.g. Frisvad et al., 1987), although in some other recent studies low levels of metabolite production have been reported (Land \& Hult, 1987). In some cases the production of a metabolite by only $30 \%$ of strains in a species has been considered a positive feature (Frisvad \& Filtenborg, 1983). The problem of loss of secondary metabolite production has been studied, especially for patulin where it was shown to involve the short halflife of critical enzymes in the pathway (Lam et al., 1988). However, this information is only available for a few metabolites. Second, distinct chemical variants occur in a number of species, and these variants were often also distinct by SEM, such as the globose conidia of the creatinepositive $P$. granulatum or the strains of $P$. camembertii with microtuberculate conidia which showed reduced physiological activity. Thirdly, there is considerable 'background' similarity amongst the fasciculate penicillia, which is supported by inter-specific fusions and serological reactions (Anné, 1986; Polonelli et al., 1986), and some strains must be considered as intermediates between species. While deteriorated strains could usually be identified as such, strain IMI 287746 (grouped in cluster 5) produced a sector which showed the properties of a different species ( $P$. camembertii, cluster 16). Studies involving a number of single conidium lines of the same strain of Penicillium have shown that there can be considerable phenotypic variation within a single strain, possibly as a result of aneuploidy (e.g. Bridge et al., 1987). It is perhaps relevant that some of the earlier workers in this genus, who recognized many more species, used strains derived from single conidia (e.g. Biourge, 1923). The role of heterokaryosis and parasexual mating has been considered in relation to variation in naturally occurring Penicillium species (e.g. Jinks, 1952), and differences seen in isozyme patterns can be due to heterokaryosis or aneuploidy (Anné \& Peberdy, 1981; Micales et al., 1986; Paterson et al., $1989 a$ ). However, these phenomena have not usually been considered in taxonomic studies.

The taxonomic results obtained in this study are generally in agreement with existing schemes (e.g. Pitt, 1980, 1986; Cruickshank \& Pitt, 1987). The main discrepancies concerning P. solitum and $P$. crustosum and $P$. olsonii and $P$. brevicompactum have already been mentioned. The one remaining discrepancy concerned the species $P$. commune which was not recovered in this study. Strains previously assigned to $P$. commune were recovered in both the $P$. solitum and $P$. aurantiogriseum clusters. The ex-type culture studied was quite different from these and further studies with another line of this culture showed it to be a typical $P$. aurantiogriseum (Bridge et al., 1989). The exact status of this species is unclear from the literature. Pitt (1980, 1986) considered this species a synonym for $P$. puberulum, which itself is now considered a synonym of $P$. aurantiogriseum. Samson \& van Reenen-Hoekstra (1988) give $P$. commune as the correct name for strains previously considered as $P$. verrucosum var. melanochlorum, a species previously considered the correct name for $P$. palitans (Samson et al., 1976). However, Frisvad (1985) considered both $P$. palitans and $P$. commune to be distinct species. The comparison between the results presented here for secondary metabolite production and the existing literature is similarly difficult due to the different strain identifications made by different authors. One example of this is the production of ochratoxin A, which has been described from $P$. viridicatum (see Turner, 1971). Subsequently, ochratoxin-producing strains have been re-identified as $P$. verrucosum, although up to $20 \%$ of the strains assigned to that species did not produce the secondary metabolite (Pitt, 1987).

This study has shown that an integrated multidisciplinary approach can be applied successfully to elucidate taxonomic problems in Penicillium. Species that were initially delineated on simple morphological features were found to be supported by critical SEM studies 
and were also determined by combinations of physiological and biochemical characters. The numerical taxonomy approach allows for a considerable number of different features to be considered together in an objective manner. This study also attempted to consider the effect on taxonomic schemes of older and atypical cultures, and the placement of these in relation to interand intra-specific variation. This has acted as a test for species concepts based on smaller numbers of characters and will therefore assist in the development of a more acceptable and stable taxonomy for this critical group. As many of the species accepted are clearly very closely related, further work is needed to fully interpret the appropriate taxonomic levels of groups within the genus; genetic and molecular studies particularly may clarify the situation further, especially with regard to strain variation and stability.

Identification matrices and keys to the fasciculate penicillia included in this study are provided in the following paper (Bridge et al., 1989).

We would like to thank Elizabeth Oliver, Penny Farnell and Devota Kavishe for their excellent technical support, and Professor E. A. Bell, Dr L. E. Fellows and the Royal Botanic Gardens, Kew for providing biochemical facilities. This work was supported by the Science and Engineering Research Council contract SO/17/84, Systematics of Microfungi of Biotechnological and Industrial Importance.

\section{REFERENCES}

ABE, S. (1956). Studies on the classification of the Penicillia. Journal of General and Applied Microbiology 2, 1-344.

ANNÉ, J. (1986) (dated 1985).* Taxonomic implication of hybridisation of Penicillium protoplasts. In Advances in Penicillium and Aspergillus Systematics, pp. 337-350. Edited by R. A. Samson \& J. I. Pitt. New York: Plenum Press.

ANNÉ, J. \& PeberdY, J. F. (1981). Characterisation of inter-species hybrids of Penicillium chrysogenum and Penicillium roquefortii by isoenzyme analysis. Transactions of the British Mycological Society 77, 401-408.

Brourge, P. (1923). Les moisissures du groupe Penicillium Link. Cellule 33, 7-331.

BRIDGE, P. D. (1985). An evaluation of some physiological and biochemical methods as an aid to the characterization of species of Penicillium subsection Fasciculata. Journal of General Microbiology 131, 1887-1895.

BRIDGE, P. D. \& HAWKSWORTH, D. L. (1984). The API ZYM testing system as an aid to the rapid identification of Penicillium isolates. Microbiological Sciences 1, 232-234.

BRIDGE, P. D. \& MAY, J. W. (1984). A numerical classification of fission yeasts of the genus Schizosaccharomyces Lindner. Journal of General Microbiology 130, 1921-1932.

Bridge, P. D., Hawksworth, D. L., Kozakiewicz, Z., Onions, A. H. S., Paterson, R. R. M. \& Sackin, M. J. (1986) (dated 1985).* An integrated approach to Penicillium systematics. In Advances in Penicillium and Aspergillus Systematics, pp. 281-309. Edited by R. A. Samson \& J. I. Pitt. New York: Plenum Press.

Bridge, P. D., Hudson, L., Kozakiewicz, Z., Onions, A. H. S. \& Paterson, R. R. M. (1987). Investigation of variation in phenotype and DNA content between single-conidium isolates of single Penicillium strains. Journal of General Microbiology 133, 995-1004.

Bridge, P. D., Hawksworth, D. L., Kozakiewicz, Z., ONIONS, A. H. S., PATERSON, R. R. M. \& SACKIN, M. J. (1989). A reappraisal of some terverticillate penicillia using biochemical, physiological and

morphological features. II. Identification. Journal of General Microbiology 135, 2967-2978.

BURNETT, J. H. (1976). Fundamentals of Mycology, 2nd edn. London: Edward Arnold.

Ciegler, A., Fennell, D. I., Sansing, G. A., Detroy, R. W. \& BeNNETT, G. A. (1973). Mycotoxin producing strains of Penicillium viridicatum: classification into subgroups. Applied Microbiology 26, 271-278.

Croft, J. H. (1987). Genetic variation and evolution in Aspergillus. In Evolutionary Biology of the Fungi, pp. 311-323. Edited by A. D. M. Rayner, C. M. Brasier \& D. Moore. Cambridge: Cambridge University Press.

Cruickshank, R. H. \& Pitt, J. I. (1987). Identification of species in Penicillium subgenus Penicillium by enzyme electrophoresis. Mycologia 79, 614-620.

Davis, N. D., Diener, U. L. \& Eldridge, D. W. (1966). Production of aflatoxins $B_{1}$ and $G_{1}$ by Aspergillus flavus in a semisynthetic medium. Applied Microbiology 14, 378-380.

DierCKX, R. P. (1901). Un essai de revision du genre Penicillium Link. Annales de la Société Scientifique de Bruxelles 25, 83-88.

El-Banna, A. A., Pitt, J. I. \& Leistner, L. (1987). Production of mycotoxins by Penicillium species. Systematic and Applied Microbiology 10, 42-46.

Fassatiova, O. (1977). A taxonomic study of Penicillium series Expansa Thom emend. Fassatiova. Acta Universitatis Carolinae-Biologica 1974, 283-335.

Frisvad, J. C. (1981). Physiological criteria and mycotoxin production as aids in identification of common asymmetric penicillia. Applied and Environmental Microbiology 41, 568-579.

Frisvad, J. C. (1985). Creatine sucrose agar, a differential medium for mycotoxin producing terverticillate Penicillium species. Letters in Applied Microbiology 1, 109-113.

FRISVAD, J. C. (1986) (dated 1985). ${ }^{*}$ Profiles of primary and secondary metabolites of value in classification of Penicillium viridicatum and related species. In Advances in Penicillium and Aspergillus Systematics, pp. 311-325. Edited by R. A. Samson \& J. I. Pitt. New York: Plenum Press. 
Frisvad, J. C. \& Filtenborg, O. (1983). Classification of terverticillate penicillia based on profiles of mycotoxins and other secondary metabolites. Applied and Environmental Microbiology 46, 13011310.

Frisvad, J. C., Filtenborg, O. \& Wicklow, D. T. (1987). Terverticillate penicillia isolated from underground seed caches and cheek pouches of bannertailed kangaroo rats (Dipodomys spectabilis). Canadian Journal of Botany 65, 765-773.

Gower, J. C. (1966). Some distance properties of latent root and vector methods used in multivariate analysis. Biometrika 53, 325-338.

HawksworTH, D. L. (1986) (dated 1985).* The typification and citation of the generic name Penicillium. In Advances in Penicillium and Aspergillus Systematics, pp. 3-7. Edited by R. A. Samson \& J. I. Pitt. New York: Plenum Press.

JINKS, J. L. (1952). Heterocaryosis in wild Penicillium. Hereditary 6, 77-87.

JoNES, D. \& SACKIN, M. J. (1980). Numerical methods in the classification and identification of bacteria with especial reference to the Enterobacteriaceae. In Microbiological Classification and Identification, pp. 73-106. Edited by M. Goodfellow \& R. G. Board. London: Academic Press.

Kendrick, W. B. \& Proctor, J. R. (1964). Computer taxonomy in the fungi imperfecti. Canadian Journal of Botany 42, 65-88.

Klittich, C. J. R., Correll, J. C. \& Leslie, J. F. (1988). Inheritance of sectoring frequency in Fusarium moniliforme (Gibberella fujikuroi). Experimental Mycology 12, 289-294.

KozAKIEwICZ, Z. (1989). Ornamentation types of conidia and conidiogenous structures in fasciculate Penicillium species, using scanning electron microscopy. Botanical Journal of the Linnean Society 99, 273-293.

LaM, K. S., Neway, J. O. \& Gaucher, G. M. (1988). In vitro stabilization of 6-methylsalicylic acid synthetase from Penicillium urticae. Canadian Journal of Microbiology 34, 30-37.

LAND, C. J. \& HulT, K. (1987). Mycotoxin production by some wood-associated Penicillium spp. Letters in Applied Microbiology 4, 41-44.

LINK, H. F. (1809). Observations in ordines plantarum naturales. Magazin Gesellschaft Naturforschender Freunde zu Berlin 3, 1-42.

Micales, J. A., Bonde, M. R. \& Peterson, G. L. (1986). The use of isozyme analysis in fungal taxonomy and genetics. Mycotaxon 27, 405-449.

MinTER, D. W., HaWksworth, D. L., ONIONS, A. H. S. \& KoZAKIEWICZ, Z. (1986) (dated 1985).* Descriptive terminology of the conidogenous structures in Aspergillus and Penicillium. In Advances in Penicillium and Aspergillus Systematics, pp. 71-87. Edited by R. A. Samson \& J. I. Pitt. New York: Plenum Press.

Mordue, J. E. M., Currah, R. S. \& Bridge, P. D. (1989). An integrated approach to Rhizoctonia taxonomy: cultural, biochemical and numerical techniques. Mycological Research 92, 78-90.

Mueller, G. M. (1985). Numerical taxonomic analyses on Laccaria (Agaricales). Mycologia 77, 121-129.

Onions, A. H. S., BRidge, P. D. \& Paterson, R. R. M.
(1984). Problems and prospects for the taxonomy of Penicillium. Microbiological Sciences 1, 185-189.

Paterson, R. R. M. (1986). Standardized one and twodimensional thin-layer chromatographic methods for the identification of secondary metabolites in Penicillium and other fungi. Journal of Chromatography 368, 249-264.

Paterson, R. R. M., Bridge, P. D., Crosswatte, M. J. \& HAWKSWORTH, D. L. (1989). A reappraisal of the terverticillate penicillia using biochemical, physiological and morphological features. III. An evaluation of pectinase and amylase isoenzymes for species characterization. Journal of General Microbiology 135, 2979-2991.

Paterson, R. R. M., KING, G. \& BRIDGe, P. D. (1990). High resolution melting curves on DNA from 14 Penicillium strains. Mycological Research (in the Press).

PITT, J. I. (1973). An appraisal of identification methods for Penicillium species: novel taxonomic criteria based on temperature and water relations. Mycologia 65, 1135-1157.

PitT, J. I. (1980) (dated 1979). + The Genus Penicillium and its Teleomorphic States Eupenicillium and Talaromyces. London: Academic Press.

PITT, J. I. (1986). A Laboratory Guide to Common Penicillium Species. North Ryde, Australia: CSIRO Division of Food Research.

PITT, J. I. (1987). Penicillium viridicatum, Penicillium verrucosum, and production of ochratoxin A. Applied and Environmental Microbiology 53, 266-269.

PITT, J. I. \& HAWKsworTH, D. L. (1986) (dated 1985).* The naming of chemical variants in Penicillium and Aspergillus. In Advances in Penicillium and Aspergillus Systematics, pp. 89-91. Edited by R. A. Samson \& J. I. Pitt. New York: Plenum Press.

Polonelli, L., Castagnola, M., D'Urso, C. \& MoRACE, G. (1986) (dated 1985).* Serological approaches for identification of Aspergillus and Penicillium species. In Advances in Penicillium and Aspergillus Systematics, pp. 267-280. Edited by R. A. Samson \& J. I. Pitt. New York: Plenum Press.

Polonelli, L., Morace, G., Rosa, R., Castagnola, M. \& Frisvad, J. C. (1987). Antigenic characterization of Penicillium camembertii and related common cheese contaminants. Applied and Environmental Microbiology 53, 872-878.

RAPER, K. B. \& THOM, C. (1949). A Manual of the Penicillia. Baltimore: Williams \& Wilkins.

SACKIN, M. J. (1981). Vigour and pattern as applied to multistate quantitative characters in taxonomy. Journal of General Microbiology 122, 247-254.

SACKIN, M. J. (1985). Comparisons of classifications. In Computer-assisted Bacterial Systematics, pp. 2136. Edited by M. Goodfellow, D. Jones \& F. G. Priest. London: Academic Press.

Samson, R. A. \& Gams, W. (1984). The taxonomic situation in the hyphomycete genera Penicillium, Aspergillus and Fusarium. Antonie van Leeuwenhoek 50, 815-824.

SAMSON, R. A. \& VAN REENEN-Hoekstra, E. S. (1988). Introduction to Food-borne Fungi. Baarn: Centraalbureau voor Schimmelcultures.

Samson, R. A., Stolk, A. C. \& Hadlok, R. (1976). Revision of the subsection fasciculata of Penicillium 
and some allied species. Studies in Mycology Baarn 11, 1-47.

Samson, R. A., Hadlok, R. \& Stolk, A. C. (1977). A taxonomic study of the Penicillium chrysogenum series. Antonie van Leeuwenhoek 43, 169-175.

Seifert, K. A. \& Samson, R. A. (1986) (dated 1985).* The genus Coremium and the synnematous penicillia. In Advances in Penicillium and Aspergillus Systematics, pp. 143-154. Edited by R. A. Samson \& J. I. Pitt. New York: Plenum Press.

SMITH, G. (1949). The effect of adding trace elements to Czapek Dox medium. Transactions of the British Mycological Society 32, 280-283.

Smith, D. \& Onions, A. H. S. (1983). The Preservation and Maintenance of Living Fungi. Kew: Commonwealth Mycological Institute.

SNeATH, P. H. A. (1979). BasiC program for a significance test for clusters in UPGMA dendrograms obtained from squared euclidean distances. Computers and Geosciences 5, 127-137.

SNEATH, P. H. A. (1985). Future of numerical taxonomy. In Computer-assisted Bacterial Systematics, pp. 415-431. Edited by M. Goodfellow, D. Jones \& F. G. Priest. London: Academic Press.
SNeath, P. H. A. \& Sokal, R. R. (1973). Numerical Taxonomy. San Francisco: W. H. Freeman.

SöDERSTRÖM, B. \& FRISVAD, J. C. (1984). Separation of closely related asymmetric penicillia by pyrolysis gas chromatography and mycotoxin production. Mycologia 76, 408-419.

StorCK, R. \& Alexopoulos, C. J. (1970). Deoxyribonucleic acid of fungi. Bacteriological Reviews 34, 126154.

StRöM, G. B. (1986). Cross-point determination of Penicillium conidia - characterization of closely related species. Journal of Applied Bacteriology 60, 557-561.

Tном, C. (1930). The Penicillia. London: Baillière, Tindall \& Cox.

TURNER, W. B. (1971). Fungal Metabolites. London: Academic Press.

Westling, R. (1911). Über die grünen Spezies der Gattung Penicillium. Arkiv für Botanik 11, 1-156.

Williams, S. T., Goodfellow, M., Alderson, G., Wellington, E. M. H., Sneath, P. H. A. \& Sackin, M. J. (1983). Numerical classification of Streptomyces and related genera. Journal of General Microbiology 129, 1743-1813.

* This book, although dated 1985, was not available until 1986.

† This book, although dated 1979, was not available until 1980 . 